\title{
Hubble Space Telescope Spectrum of SN 1987A at an Age of 8 Years: Radioactive Luminescence of Cool Gas
}

\section{Citation}

Chugai, Nikolai N., Roger A. Chevalier, Robert P. Kirshner, and Peter M. Challis. 1997. "Hubble Space TelescopeSpectrum of SN 1987A at an Age of 8 Years: Radioactive Luminescence of Cool Gas." The Astrophysical Journal 483 (2): 925-40. https://doi.org/10.1086/304253.

\section{Permanent link}

http://nrs.harvard.edu/urn-3:HUL.InstRepos:41399772

\section{Terms of Use}

This article was downloaded from Harvard University's DASH repository, and is made available under the terms and conditions applicable to Other Posted Material, as set forth at http:// nrs.harvard.edu/urn-3:HUL.InstRepos:dash.current.terms-of-use\#LAA

\section{Share Your Story}

The Harvard community has made this article openly available. Please share how this access benefits you. Submit a story.

Accessibility 
THE Astrophysical Journal, 483:925-940, 1997 July 10

(C) 1997. The American Astronomical Society. All rights reserved. Printed in U.S.A.

\title{
HUBBLE SPACE TELESCOPE SPECTRUM OF SN 1987A AT AN AGE OF 8 YEARS: RADIOACTIVE LUMINESCENCE OF COOL GAS
}

\author{
Nikolai N. Chugai, ${ }^{1,2}$ Roger A. Chevalier, ${ }^{1}$ Robert P. Kirshner, ${ }^{3}$ and Peter M. Challis ${ }^{3}$ \\ Received 1996 May 3; accepted 1997 February 11
}

\begin{abstract}
The Hubble Space Telescope 2000-8000 ̊ spectrum of SN 1987A observed on 1995 January 7 (7.87 yr after the explosion) is dominated by $\mathrm{H} \alpha$ and UV lines, including $\mathrm{Mg}$ II-Mg I $\lambda 2825$ (equal to $\mathrm{H} \alpha$ in luminosity), Fe II UV 2 (two-thirds the intensity of $\mathrm{H} \alpha$ ), $\mathrm{Fe}$ II UV 3 (one-half the intensity of $\mathrm{H} \alpha$ ), and a $3730 \AA$ A emission feature identified with a blend of [O II] $\lambda 3727$ and $\mathrm{Fe}$ I emission lines. [O I] $\lambda 6300$ and lines of $[\mathrm{Ca} \mathrm{II}]$ and $\mathrm{Na}$, as well as some $\mathrm{Fe}$ II optical forbidden and permitted lines are present at visual wavelengths. Also present are a number of weak emission features, which are presumably metal lines produced by photon degradation as a result of reprocessing of UV radiation into metal lines. Modeling the $\mathrm{Mg}$ II-Mg I lines provides the velocity of the outer visible radius of the envelope, $9000 \pm 500 \mathrm{~km} \mathrm{~s}^{-1}$ in the Mg II $\lambda 2800$ line, which is consistent with the earlier direct HST imaging at near-UV wavelengths. The UV/optical emission lines originate from the radioactive luminescence of the cool gas $(T \approx 130-160$ $\mathrm{K})$. The metal lines reflect the instantaneous reprocessing of the energy deposited from ${ }^{44} \mathrm{Ti}$ radioactive decays through collisions with fast electrons, while the $\mathrm{H} \alpha$ emission primarily comes from the recombination of previously ionized hydrogen. The overall luminosity of the Fe II emission lines, $\sim 10^{35}$ ergs $\mathrm{s}^{-1}$, can be explained if the bulk of the positrons from a mass (1-2) $\times 10^{-4} M_{\odot}$ of ${ }^{44} \mathrm{Ti}$ release their energy in the iron-rich material, which suggests the presence of a magnetic field $B>5 \times 10^{-13} \mathrm{G}$ prohibiting the escape of positrons into oxygen and hydrogen gas. The ionized fraction in the iron-rich material is small $(0.2-0.3)$, and the total UV/optical emission from $\mathrm{Fe}$ I should be comparable to that from Fe II. Most of the $10^{36} \mathrm{ergs} \mathrm{s}^{-1}$ deposited by the ${ }^{44} \mathrm{Ti}$ positrons should be emitted in the $\mathrm{Fe}$ II 26 $\mu \mathrm{m}$ line. The observed $\mathrm{H} \alpha$ luminosity decrease, by 5 orders of magnitude between the ages of 1 to $8 \mathrm{yr}$, is reproduced in a time-dependent model of ionization and cooling with the "standard" amount of radioactive nuclides. However, an additional source of energy at the present epoch with a deposition rate 30 ergs $\mathrm{s}^{-1} \mathrm{~g}^{-1}\left(\approx 10^{36} \mathrm{ergs} \mathrm{s}^{-1}\right.$ in the whole envelope $)$ is not ruled out. The present average temperature in the hydrogen envelope predicted by the time-dependent model is $130 \mathrm{~K}$, which is lower than the value $T \approx 350 \mathrm{~K}$ obtained from the observed Balmer continuum shape. However, the shape is affected by a possible contribution of metal lines to the Balmer continuum. The luminosity of the $\left[\mathrm{O}_{\mathrm{I}}\right] \lambda 6300$ doublet is consistent with that expected for the deposited energy of $\gamma$-rays from (1-2) $\times 10^{-4} M_{\odot}$ of ${ }^{44} \mathrm{Ti}$ for an assumed 1.5-2 $M_{\odot}$ of oxygen. If the oxygen mass does not exceed $2 M_{\odot}, 1 \times 10^{-4} M_{\odot}$ is a lower limit for the amount of ${ }^{44} \mathrm{Ti}$ in SN 1987A. The maximum fraction of the ${ }^{44} \mathrm{Ti}$ positron energy deposited into oxygen-rich material does not exceed $5 \%$, which is consistent with positron trapping in Fe-rich material. The [O I $] 26300$ line intensity rules out the presence of a central source of $\gamma$-radiation $(h v>100 \mathrm{keV})$ with a luminosity $L_{\gamma}>4 \times 10^{36} \mathrm{ergs} \mathrm{s}^{-1}$.

Subject headings: line: formation - nuclear reactions, nucleosynthesis, abundances -
\end{abstract} supernovae: individual (SN 1987A)

\section{INTRODUCTION}

Since the explosion, SN 1987A has passed through several phases characterized by different sources of energy and different physical processes for the production of the observed radiation spectrum. The physical processes relevant to the first 4-5 yr of the supernova evolution were reviewed by McCray (1993). After the photospheric epoch ( $t<120$ days), dominated by the thermal energy of trapped radiation and ${ }^{56} \mathrm{Co}$ decay, the supernova entered the nebular phase, powered by ${ }^{56} \mathrm{Co}$ decay, which was overtaken by ${ }^{57}$ Co decay after 1200 days and then $(t>1500$ days) by ${ }^{44} \mathrm{Ti}$ decay. The remarkable properties of the latest epoch are strong cooling of the ejecta (both the metal-rich

\footnotetext{
${ }^{1}$ Department of Astronomy, University of Virginia, P.O. Box 3818, Charlottesville, VA 22903-0818; rac5x@virginia.edu.

${ }^{2}$ Institute of Astronomy, Russian Academy of Sciences, Pyatnitskaya 48, 109017 Moscow, Russia; nchugai@ra.inasan.rssi.ru.

${ }^{3}$ Center for Astrophysics, 60 Garden Street, MS-19, Cambridge, MA; kirshner@cfa.harvard.edu, pchallis@cfa.harvard.edu.
}

core and the hydrogen envelope) down to hundreds of Kelvins and nonsteady cooling and recombination, e.g., in hydrogen (Clayton et al. 1992; Fransson \& Kozma 1993; Fransson, Houck, \& Kozma 1996). These facts mean that the ionization and thermal state of the supernova envelope is determined not only by the present energy source but also by the history of the energy release. The bolometric luminosity at an age of 3-4 yr, which is mostly radiated in the far IR, can be reproduced in a model of time-dependent cooling and ionization with the generally accepted amounts of radioactive nuclides ${ }^{56} \mathrm{Ni},{ }^{57} \mathrm{Ni}$, and ${ }^{44} \mathrm{Ti}$ (Fransson \& Kozma 1993). This implies that an additional source for ionization and heating, such as an embedded pulsar or an accretion-powered source, is not needed to explain the data.

Although far-IR radiation is predicted to dominate the radiative output, it is not accessible to present-day observations. Optical and UV radiation remain important diagnostic tools for probing the energy source and the structure of the envelope, as demonstrated by the analysis of previously obtained UV and optical spectra of SN 1987A (Pun 
et al. 1995). Yet this subject is poorly understood because SN 1987A, at its present stage, is in many respects a unique object. A basic question is whether the present-day UV/ optical spectrum of SN 1987A can be understood in terms of straightforward physical processes in the expanding envelope with the conventional parameters and amounts of radioactive nuclides. We know that a compact object has formed in SN 1987A from the observed neutrino burst (Arnett et al. 1989) and there may be energetic processes associated with it at the present time that affect the UV/ optical spectrum.

These issues are addressed in the present paper. The observational data used are the spectrum of SN 1987A obtained by the Hubble Space Telescope (HST) on 1995 January 7, nearly 8 yr after the explosion, which took place on 1987 February 23. This is the first spectrum of SN 1987A obtained after the COSTAR repair, which improved the image quality and the photometric reliability of Faint Object Spectrograph (FOS) observations. We build on the work of Wang et al. (1996), who discussed HST spectra of SN 1987A taken in 1992 and 1993. In $\S 2$, we present the basic observational parameters. In $\S 3$, we present plausible identifications for the major spectral lines, which in several cases turned out to have ambiguous identifications. In $\S 4$, we use established models of the density and composition structure of SN 1987A to interpret the heavy element line emission. Because the expanding gas is substantially opti- cally thin in the continuum, we use a nebular analysis. In $\S 5$, we emphasize the formation of the $\mathrm{H} \alpha$ line and its luminosity evolution after $t=1 \mathrm{yr}$ and develop a model for the time-dependent ionization and cooling in the hydrogen-rich envelope. Our conclusions are presented in $\S 6$.

\section{OBSERVATIONS}

The spectra of SN 1987A were obtained by the HST FOS as part of the Supernovae INtensive Study (SINS) project. The observations were obtained on 1995 January 7-8 in the post-COSTAR era. The spectrum covers the range of 1650 $9200 \AA$ A. A detailed description of the instrumentation can be found in the FOS Instrument Handbook (Keyes et al. 1995). All the data were reduced using the routine HST postprocessing pipeline system maintained by the Space Telescope Science Institute (Koratkar 1994).The most recent calibration data were used in the postprocessing, as recommended by the FOS instrument scientists. All the data were taken with the FOS Red Side through the circular 0.5 aperture. Figure 1 shows the location of the aperture relative to the debris, inner ring, and outer loop structures. The earlier spectra taken by the SINS project (Wang et al. 1996) were taken with a rectangular slit $0.25 \times 2.0$, and included the spectra of the debris and the ring simultaneously. In that case, the spectral resolution of the FOS allowed us to separate the broad lines of the debris and the narrow lines of the ring spectra. After COSTAR was deployed, the scale

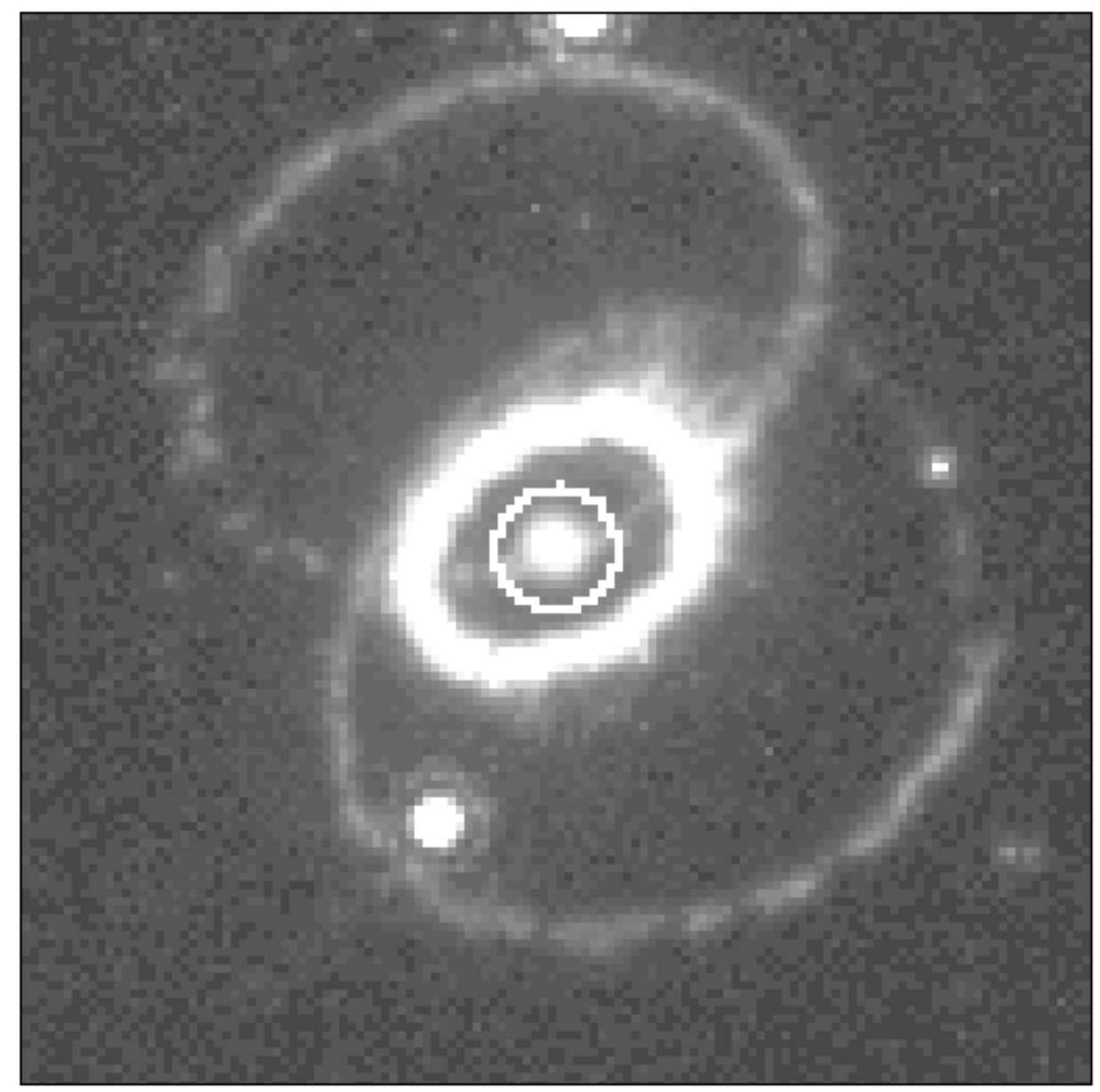

FIG. 1.-Position of the circular aperture of the FOS superimposed on the HST image of SN 1987A. The inner and outer rings surrounding the supernova are visible. 


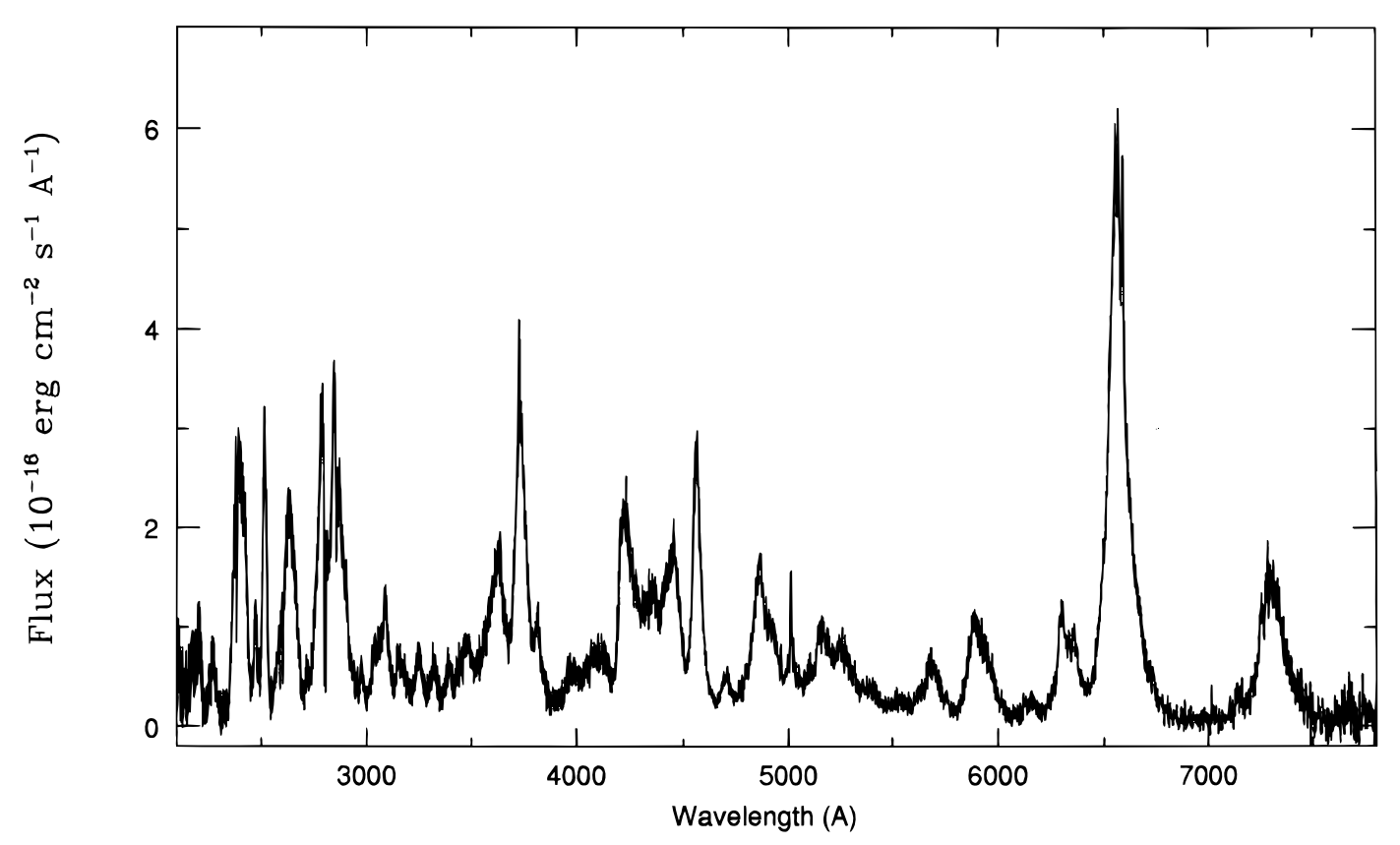

FIG. 2.- $-H S T$ spectrum of SN 1987 A on 1995 January 7

of the FOS diodes changed from 1".7 to 1"3. The scale change and our need to make observations at a specific orientation led to a change in our observing strategy. We now take separate spectra of the debris and the circumstellar ring with the 0.5 circular aperture. The complete spectrum of the debris is shown in Figure 2.

After COSTAR the FOS target acquisition improved. The dominant error in FOS spectrophotometry is the error in the location of the target in the aperture. The improved centering of SN 1987A within the aperture improves the flux calibration over that achieved in the previous cycles. The absolute flux calibration is good to about $5 \%$, while the earlier data had uncertainties of about $20 \%$ in the UV and $10 \%$ in the optical. From Figure 1 it is clear that the northern outer loop passes through the small circular aperture. The spectra have narrow features that we attribute to the outer loop (Panagia et al. 1996). Future observations with the FOS will be comparable to these 1995 data and will extend into the far UV, 1150-1650 ̊. Table 1 contains the summary of the observations, including data-set names, grating, wavelength coverage, exposure time, and resolution.

\section{LINE FLUXES AND IDENTIFICATIONS}

The spectrum at the present epoch is primarily composed of emission lines, which were mostly identified and discussed by Wang et al. (1996). In what follows, we treat spectral-line evolution (if any) and some ambiguous points of particular identifications. The UV part of the spectrum is especially complex because of radiative transfer effects and we list only a few plausible identifications in that region. The strongest emission features in the spectrum are listed in Table 2, with identifications, fluxes, and luminosities. Correction for Galactic absorption corresponds to $E(B-V)=0.2 \mathrm{mag}$ (Panagia et al. 1987) and the extinction law from Seaton (1979). The integrated dereddened flux from SN 1987A in the $1600-8500 \AA$ range is $1.2 \times 10^{-12}$ ergs $\mathrm{s}^{-1} \mathrm{~cm}^{-2}$, which results in a total UV/optical luminosity in this wavelength range of $3.5 \times 10^{35} \mathrm{ergs} \mathrm{s}^{-1}$ (for the adopted distance of $50 \mathrm{kpc}$ ). The integrated luminosity of the quasi-continuum in this wavelength range is about $7.3 \times 10^{34} \mathrm{ergs} \mathrm{s}^{-1}$.

Hydrogen.-The $\mathrm{H} \alpha$ emission is still relatively strong, although the $2825 \AA$ feature (corrected for absorption) is

TABLE 1

SUMMARY OF FOS OBSERVATIONS OF SN 1987A

\begin{tabular}{ccccc}
\hline \hline Data Set & Grating & $\begin{array}{c}\text { Wavelength Coverage } \\
(\AA)\end{array}$ & $\begin{array}{c}\text { Exposure Time } \\
(\mathrm{s})\end{array}$ & $\begin{array}{c}\text { Resolution FWHM } \\
(\AA)\end{array}$ \\
\hline y2hw0103t ...... & G190H & $1650-2310$ & 4000 & 1.35 \\
y2hw0104t ..... & G190H & $1650-2310$ & 2000 & 1.35 \\
y2hw0105t ...... & G270H & $2223-3278$ & 1700 & 1.90 \\
y2hw0106t ..... & G270H & $2223-3278$ & 2300 & 1.90 \\
y2hw0107t ...... & G400H & $3240-4784$ & 1700 & 2.79 \\
y2hw0108t ...... & G400H & $3240-4784$ & 2300 & 2.79 \\
y2hw0109t ...... & G570H & $4571-6817$ & 1500 & 4.06 \\
y2hw010at ...... & G570H & $4571-6817$ & 2500 & 4.06 \\
y2hw010bt ..... & G780H & $6281-9219$ & 1200 & 6.17 \\
y2hw010ct ...... & G780H & $6281-9219$ & 2800 & 6.17 \\
\hline
\end{tabular}


TABLE 2

EMISSION LINES IN THE SN 1987A SPECTRUM ON 1995 JANUARY 7

\begin{tabular}{|c|c|c|c|c|c|}
\hline $\begin{array}{l}\lambda_{\text {gbs }} \\
(\AA)\end{array}$ & $\begin{array}{l}\lambda_{\text {gor }} \\
(\AA)\end{array}$ & $\begin{array}{c}\text { FWHM } \\
(\AA)\end{array}$ & $\begin{array}{c}F \\
\left(10^{-14} \mathrm{ergs} \mathrm{cm}^{-2}\right. \\
\left.\mathrm{s}^{-1}\right)\end{array}$ & $\begin{array}{c}F^{\mathrm{cor}} \\
\left(10^{-14} \mathrm{ergs} \mathrm{cm}^{-2}\right. \\
\left.\mathrm{s}^{-1}\right)\end{array}$ & Identification \\
\hline $1668.7 \ldots \ldots$ & 1667.11 & 52.4 & 0.495 & 2.09 & \\
\hline $1773.2 \ldots \ldots$ & 1771.51 & 7.4 & 0.082 & 0.35 & \\
\hline $1824.8 \ldots \ldots$ & 1823.06 & 47.1 & 0.358 & 1.53 & \\
\hline $1908.5 \ldots \ldots$ & 1906.68 & 10.7 & 0.124 & 0.56 & \\
\hline $1979.6 \ldots \ldots$ & 1977.71 & 30.1 & 0.235 & 1.16 & \\
\hline $2101.2 \ldots \ldots$ & 2099.20 & 41.0 & 0.122 & 0.72 & \\
\hline $2206.3 \ldots \ldots$ & 2204.20 & 32.6 & 0.295 & 1.76 & \\
\hline $2271.5 \ldots \ldots$ & 2269.38 & 40.4 & 0.138 & 0.75 & \\
\hline $2399.0 \ldots \ldots$ & 2396.71 & 61.8 & 1.50 & 6.54 & Fe II UV 2 \\
\hline $2472.6 \ldots \ldots$ & 2470.24 & 24.6 & 0.212 & 0.83 & \\
\hline $2515.5 \ldots \ldots$ & 2513.10 & 22.4 & 0.617 & 2.31 & Si I UV $1 ?$ \\
\hline $2632.4 \ldots \ldots$ & 2629.89 & 68.9 & 1.38 & 4.66 & Fe II UV 1 \\
\hline $2717.3 \ldots \ldots$ & 2714.71 & 23.9 & 0.070 & 0.22 & \\
\hline $2821.4 \ldots \ldots$ & 2818.71 & 111.2 & 3.25 & 9.81 & $\mathrm{Mg}_{\text {II }} \lambda 2800, \mathrm{Mg}_{\text {I }} \lambda 2852$ \\
\hline $2975.3 \ldots \ldots$ & 2972.46 & 23.3 & 0.088 & 0.25 & \\
\hline $3073.9 \ldots \ldots$ & 3070.97 & 39.8 & 0.594 & 1.62 & \\
\hline $3150.1 \ldots \ldots$ & 3147.10 & 79.7 & 0.199 & 0.53 & \\
\hline $3248.2 \ldots \ldots$ & 3245.10 & 35.0 & 0.176 & 0.46 & \\
\hline $3320.0 \ldots \ldots$ & 3316.83 & 32.9 & 0.122 & 0.31 & \\
\hline $3390.2 \ldots \ldots$ & 3386.97 & 31.3 & 0.132 & 0.33 & \\
\hline $3481.3 \ldots \ldots$ & 3477.98 & 99.1 & 0.553 & 1.36 & \\
\hline $3622.2 \ldots \ldots$ & 3618.75 & 89.5 & 1.27 & 3.03 & \\
\hline $3734.8 \ldots \ldots$ & 3731.24 & 53.0 & 1.63 & 3.93 & {$\left[\mathrm{O}_{\mathrm{I}}\right] \lambda 3727, \mathrm{Fe}_{\mathrm{I}}$ (multiplets 5 and 21 ) } \\
\hline $3806.3 \ldots \ldots$ & 3802.67 & 58.3 & 0.371 & 0.86 & \\
\hline $3975.2 \ldots \ldots$ & 3971.41 & 65.5 & 0.167 & 0.38 & $\mathrm{Ca}$ II \\
\hline $4100.4 \ldots \ldots$ & 4096.49 & 135.4 & 0.558 & 1.23 & \\
\hline $4227.0 \ldots \ldots$ & 4222.97 & 59.4 & 1.02 & 2.21 & {$[\mathrm{Fe}$ II] $\lambda 4244(21 \mathrm{~F})$} \\
\hline $4343.4 \ldots \ldots$ & 4339.26 & 48.9 & 1.79 & 3.82 & {$[\mathrm{Fe} \mathrm{II}] \lambda 4359(7 \mathrm{~F})$} \\
\hline $4457.2 \ldots \ldots$ & 4452.95 & 68.9 & 0.86 & 1.80 & {$[\mathrm{Fe}$ II $] \lambda 4458(6 \mathrm{~F})$} \\
\hline $4563.8 \ldots \ldots$ & 4559.45 & 45.8 & 1.16 & 2.40 & $\mathrm{Mg} \mathrm{I}] \lambda 4571$ \\
\hline $4702.3 \ldots \ldots$ & 4697.82 & 50.3 & 0.142 & 0.29 & {$[\mathrm{Fe}$ II $] \lambda 4728(4 \mathrm{~F})$} \\
\hline $4861.0 \ldots \ldots$ & 4856.37 & 33.7 & 1.38 & 2.71 & $\mathrm{H} \beta,[\mathrm{Fe} \mathrm{II}] \lambda 4889$ \\
\hline $5011.6 \ldots \ldots$ & 5006.82 & 26.3 & 0.317 & 0.61 & Fe II $\lambda 5018,[\mathrm{O}$ III $] \lambda 5007$ \\
\hline $5163.5 \ldots \ldots$ & 5158.58 & 73.5 & 0.536 & 1.01 & {$[\mathrm{Fe}$ II $] \lambda 5159(19 \mathrm{~F}), \mathrm{Fe}_{\text {II }} \lambda 5169$} \\
\hline $5261.4 \ldots \ldots$ & 5256.38 & 89.4 & 0.486 & 0.90 & {$[\mathrm{Fe}$ II $] \lambda 5261$ (19F) } \\
\hline $5685.6 \ldots \ldots$ & 5680.18 & 109.5 & 0.485 & 0.85 & {$[\mathrm{Fe} \mathrm{I}] \lambda 5696(2 \mathrm{~F}) ?$} \\
\hline $5889.7 \ldots \ldots$ & 5884.09 & 29.6 & 1.05 & 1.78 & $\mathrm{Na}$ I $\lambda 5892$ \\
\hline $6153.8 \ldots \ldots$ & 6147.93 & 80.0 & 0.102 & 0.17 & \\
\hline $6355.0 \ldots \ldots$ & 6348.94 & 15.1 & 1.08 & 1.74 & {$\left[\mathrm{O}_{\mathrm{I}}\right] \lambda 6300,6364$} \\
\hline $6568.6 \ldots \ldots$ & 6562.34 & 63.2 & 6.57 & 10.4 & $\mathrm{H} \alpha$ \\
\hline $7148.2 \ldots \ldots$ & 7141.39 & 45.9 & 0.128 & 0.19 & {$[\mathrm{Fe}$ II $] \lambda 7155(14 \mathrm{~F})$} \\
\hline $7306.3 \ldots \ldots$ & 7299.33 & 136.4 & 2.02 & 3.00 & {$[\mathrm{Ca}$ II $] \lambda 7302$} \\
\hline
\end{tabular}

now dominant. In addition to the recombination lines $\mathrm{H} \alpha$ and $\mathrm{H} \beta$, the spectrum shows an emission feature at $3600 \AA$ that can be identified with the low-temperature Balmer continuum found in an earlier HST spectrum of SN 1987A at the age of $5.1 \mathrm{yr}$ (Wang et al. 1996). At $t=6 \mathrm{yr}$, the Paschen continuum from gas with a low temperature $(T \approx 200 \mathrm{~K})$ was also observed (Chugai et al. 1993).

Taking the recombination emissivity dependence on velocity from the $\mathrm{H} \alpha$ profile and assuming an isothermal envelope, we find that the Balmer continuum profile implies $T_{e}=350 \mathrm{~K}$ (Fig. 3). For this simple model, using the recombination coefficients of Martin (1988), the Balmer continuum luminosity is a factor of 0.73 times that predicted by the $\mathrm{H} \alpha$ luminosity; the flux of the best-fit model in Figure 3 is multiplied by this factor. The possible error in the temperature determination with a constant, smooth continuum is $\pm 100 \mathrm{~K}$. The error must be larger than this value because we do not know the shape of the continuum. The uncertainty in the continuum level, the possible contribution of other emission lines, and the luminosity disagreement make the temperature determination from the Balmer continuum unreliable. Yet the modeling shows that the cool Balmer continuum should be the major constituent of the $3600 \AA$ emission feature.

The HST observation at the previous epoch (2210 days) shows the presence of the Balmer continuum with a temperature of $400 \mathrm{~K}$ (Wang et al. 1996), which corresponds to $200 \mathrm{~K}$ at the present epoch if there is only adiabatic cooling $\left(T \propto t^{-2}\right)$. This value is significantly lower than our best-fit value of $350 \mathrm{~K}$, but is tolerable given the uncertainties mentioned above.

The Balmer decrement, corrected for reddening, is $I(\mathrm{H} \alpha) / I(\mathrm{H} \beta)=3.8$. The recombination theory in case B predicts a decrement of 4.5 for a temperature of $350 \mathrm{~K}$ (Martin 1988). The presence of other lines contributing to the measured $\mathrm{H} \beta$ flux may be the cause of this discrepancy. The two-photon hydrogen continuum is expected to contribute a total luminosity of 2.6 times the $\mathrm{H} \alpha$ luminosity. This corresponds to an observed flux of $1.6 \times 10^{-17} \mathrm{ergs} \mathrm{s}^{-1} \mathrm{~cm}^{-2}$ $\AA^{-1}$ at $2800 \AA$, which is a factor of 2 below the observed quasi-continuum level.

Oxygen.-The oxygen doublet [O I] $\lambda \lambda 6300,6364$ is still a significant feature in the spectrum. The excitation of this line and many other metal lines is related to collisions with 


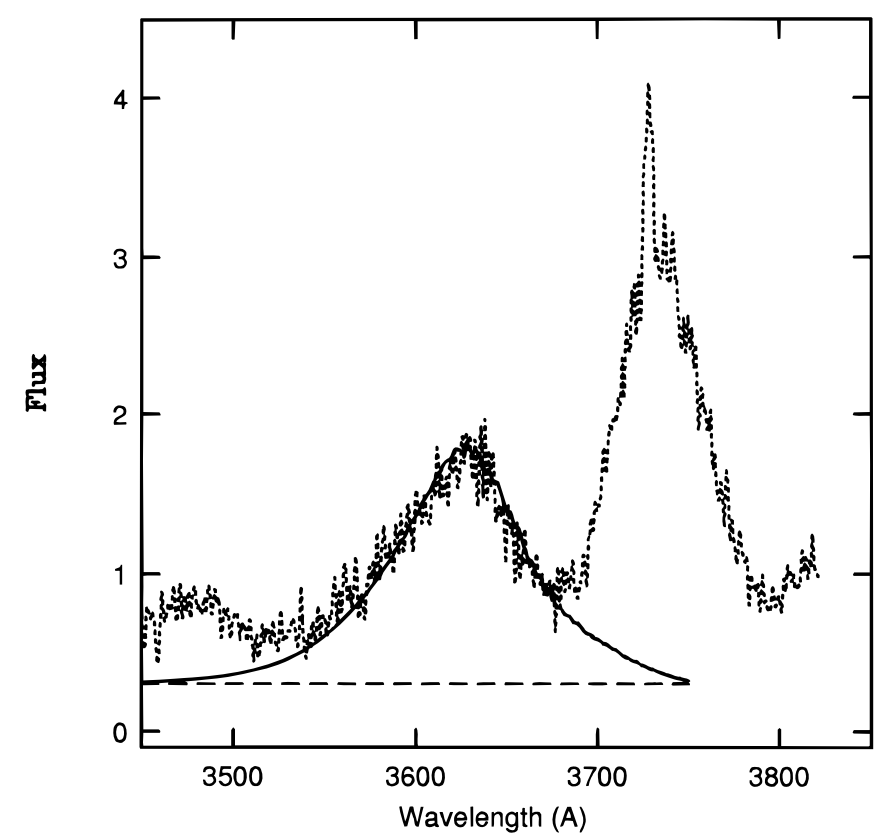

Fig. 3.-Observed Balmer continuum at $t=7.87 \mathrm{yr}$ (dotted line) compared to a model with $T=350 \mathrm{~K}$ and an emissivity distribution derived from the $\mathrm{H} \alpha$ line (solid line). The adopted continuum level is shown by the dashed line.

fast secondary electrons (Fransson \& Kozma 1993). The strong emission line at $3730 \AA$ suggests the presence of the [O II] $\lambda 3727$ doublet (Wang et al. 1996). As discussed by Wang et al., this line could be excited by fast electrons via the ionization of neutral oxygen, which leaves $\mathrm{O}$ II in an excited state. The corresponding branching ratio is relatively high (see Laher \& Gilmore 1990). The emission at $7300 \AA$ might contain some contribution of [O II] $\lambda 7324$ in addition to the $[\mathrm{Ca}$ II $] \lambda 7300$ intensity. The relative intensities of all oxygen lines excited by the fast electrons, considered in the next section, cast some doubt on the dominant contribution of [O II] to the $3730 \AA$ emission.

An additional check on the identification of the [O II] $\lambda 3727$ line is to compare the profile of the $3730 \AA$ emission with that of the $\left[\mathrm{O}_{\mathrm{I}}\right] \lambda 6300$ line. The lines [O I] $\lambda 6300$ and [O II] $\lambda 3727$ should arise from the same mass of oxygen and should therefore have similar profiles. This means that the synthetic profile of the $\left[\mathrm{O}_{\mathrm{I}}\right] \lambda 6300$ doublet, composed using the [O II] $\lambda 3727$ profile and the nebular ratio of the $\lambda 6300 / \lambda 6364$ components, should be the same as that of the observed [O I] doublet unless the electron density distribution has a strong radial dependence and collisional deexcitation is significant for the [O II] line. Figure 4 shows that the synthetic profile differs somewhat from the observed one. The major differences are the position of the maximum and the different shape of the blue half of the profile. Although a contribution of [Fe II] $\lambda \lambda 6340,6444(15 \mathrm{~F})$ is conceivable (Wang et al. 1996), such a contribution will not remove the discrepancy. We conclude that either there are other emission-line contributors to the $3730 \AA$ feature or the lines of $[\mathrm{O} \mathrm{I}]$ and $[\mathrm{O} \mathrm{II}]$ originate from different regions.

We considered alternatives to the oxygen origin of the $3730 \AA$ emission. Bearing in mind the low ionization of metals indicated by the estimates in $\S \S 4.2$ and 5.2, we suggest that lines of $\mathrm{Fe}$ I (multiplets 5 and 21) might contribute to this feature. Multiplet 5 is a resonance multiplet with

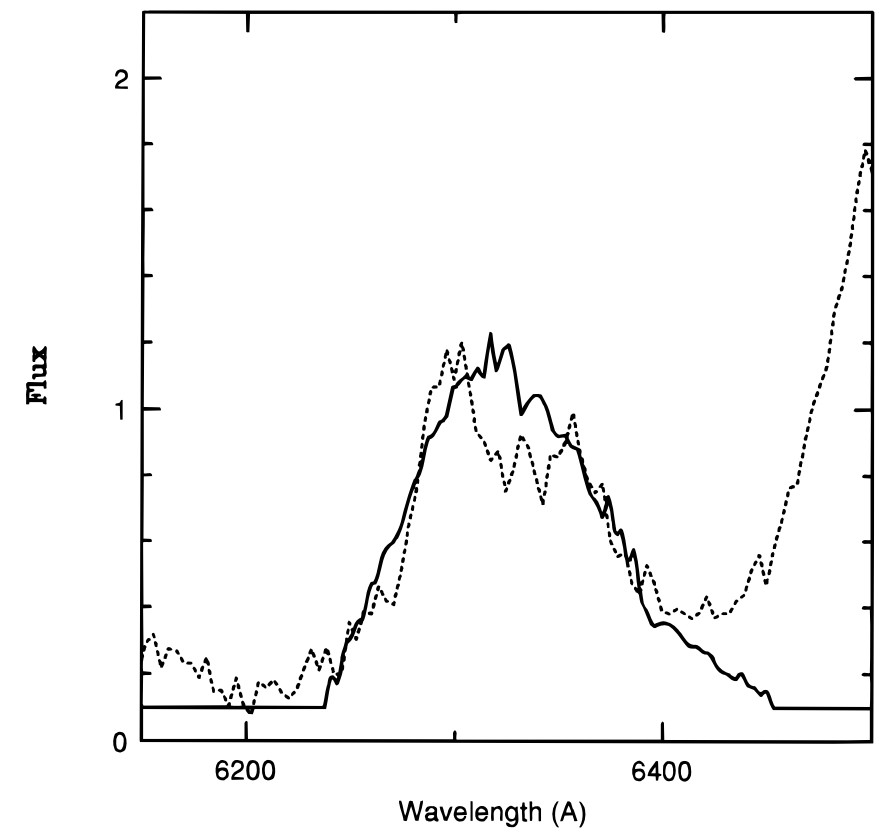

FIG. 4.-Observed emission from the oxygen doublet [O I] $\lambda \lambda 6300$, 6364 (dotted line) compared to a model synthesized from the $3730 \AA$ emission feature profile (solid line), assuming that the latter feature is the [O II] $\lambda 3727$ line.

a very low probability of conversion into the subordinate multiplet 16 . As for multiplet 21 , it is a subordinate transition and has a common upper term with the resonance multiplet UV 1 of Fe I. In order to check the possible effect of $\mathrm{Fe}$ I lines, we computed the synthetic spectrum of the $3730 \AA$ feature, taking into account all 24 lines of multiplets 5 and 21 of $\mathrm{Fe}$. The profile of each line was taken to have constant emissivity $j$ for $v<1700 \mathrm{~km} \mathrm{~s}^{-1}, j=0$ at $v>4000$ $\mathrm{km} \mathrm{s}^{-1}$, and $j \propto v^{-4}$ in the intermediate region. This emissivity profile should qualitatively reflect the velocity of the metal core $\left(\approx 1700 \mathrm{~km} \mathrm{~s}^{-1}\right)$, while the outer velocity tail rather arbitrarily describes the emissivity due to photon degradation in the outer material, which is relatively opaque in the resonance lines. The relative intensity of a given transition $u-l$ within each multiplet was assumed to be proportional to the statistical weight of the upper level $u$ and the branching ratio $A_{u 1} /\left(A_{u 1}+A_{u 2}\right)$, where $A$ is the spontaneous emission rate. This approximation is rough, but it illustrates the possibility that there is an Fe I contribution to the $3730 \AA$ feature. We found that the synthetic blend of $\mathrm{Fe}$ I lines plus a small fraction of [O II] emission qualitatively reproduces the observed feature (Fig. 5). In this particular model, $80 \%$ of the flux comes from $\mathrm{Fe}$ I lines (60\% from multiplet 5 and $20 \%$ from multiplet 21 ) and $20 \%$ of the flux comes from [O II] 23727 . Thus a substantial contribution of $\mathrm{Fe}$ I emission to the $3730 \AA$ feature is feasible, although a significant contribution of $[\mathrm{O}$ II] to the $3730 \AA$ feature cannot be ruled out. We will return to this issue in $\S 4.4$.

Sodium.-We identify the $5890 \AA$ A emission with the $\mathrm{Na}$ I doublet. He I $\lambda 5876$ is unlikely to contribute significantly to this emission because another $\mathrm{He}$ I triplet-state line at 7065 $\AA$ is absent. It is remarkable that the flux of the Na I doublet is comparable to that of the [O I] doublet. This fact seems puzzling given the expected low relative abundance of sodium: $(\mathrm{Na} / \mathrm{O})_{\odot} \approx 1 / 400$ by number. However, the large 


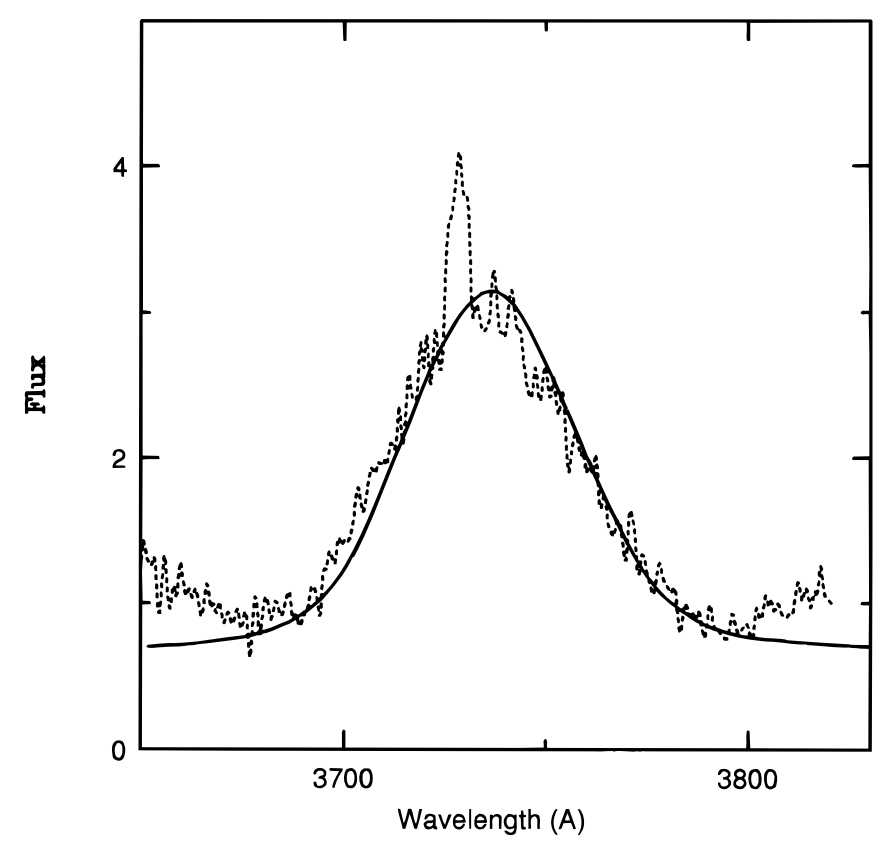

FIG. 5.-The observed emission feature at $3730 \AA$ (dotted line) modeled by the $\mathrm{Fe}$ I ( $80 \%$ of emission) and [O II] $\lambda 3727$ ( $20 \%$ of emission) lines (solid line).

cross section for $\mathrm{Na}$ I doublet excitation by nonthermal electrons, which exceeds the cross section for [O I ] doublet excitation by $\sim 10^{2}$, makes up for the difference in abundances.

Magnesium.-In addition to the $\mathrm{Mg}$ I] $\lambda 4571$ line, which was present in the spectrum after 250 days, the $H S T$ spectrum at $5.1 \mathrm{yr}$ revealed a strong emission feature between 2750 and $2900 \AA$ (Wang et al. 1996). This feature was identified as a blend of the resonance lines $\mathrm{Mg}$ I $\lambda 2852$ and $\mathrm{Mg}$ II 22800. The possible contribution of other lines to this emission feature is considered in the next section.

Calcium.-The forbidden [Ca II] $\lambda 7300$ doublet is the only Ca II feature seen in the spectrum. The absence of the Ca II $\lambda 3950$ doublet is explained by the large optical depth of the resonance doublet, which results in a low branching ratio for this line. For the same reason we expect that practically all excitations of the resonance line are deexcited through the IR triplet $\mathrm{Ca}$ II $\lambda 8600$, which must have a flux comparable with that of [Ca II] 27300 . Unfortunately, the $H S T$ data in the region of $8600 \AA$ are too noisy to detect the Ca II IR triplet.

Iron.-At the present epoch, there is no close analogy among iron-line-emitting astrophysical objects to SN 1987A, which is characterized by the nonthermal excitation of very cool gas. Some of the prominent emission features in the visible range of the spectrum, e.g., 4227,4343 , and 4457 $\AA$, are likely to be dominated by [Fe II] forbidden lines of multiplets $21 \mathrm{~F}, 7 \mathrm{~F}$, and $6 \mathrm{~F}$ (see Wang et al. 1996), while the 5163 and $5261 \AA$ lines could be identified with the Fe II multiplet 19F. Weak lines of the Fe II multiplet 42 at $\lambda 5018$ and $\lambda 5169$ seem to be present also. Some emission features could be attributed to $[\mathrm{Fe} \mathrm{I}]$ lines. The presence of $[\mathrm{Fe} \mathrm{I}]$ in the late (day 2467) spectrum of SN 1987A was suggested by Stathakis, Cannon, \& Spyromilio (1995). The emission feature at $5670 \AA$ might be identified with the strongest lines $5639 \AA$ and $5696 \AA$ of the $2 \mathrm{~F}$ multiplet of [ $[\mathrm{Fe} \mathrm{I}]$.

The strong UV emission features at $2632 \AA$ and $2399 \AA$ can be identified with the Fe II UV 1 and UV 2 multiplets respectively. Emission lines of the UV 3 multiplet of $\mathrm{Fe}$ II lie shortward of lines of UV 2 by $250-6900 \mathrm{~km} \mathrm{~s}^{-1}$ and may be effectively scattered by UV 2 lines. With this hypothesis concerning multiplet UV 3 , we conclude that all the strong UV resonance $\mathrm{Fe}$ II multiplets (UV 1, 2, and 3) are present in the spectrum of SN 1987A.

$2515 \AA$ emission line.- The prominent unidentified emission line in the UV spectrum at $2515 \AA$ may arise from a combination of the fluorescence and nonlocal radiation transfer in the "fence" of numerous optically thick metal UV resonance lines. Alternatively, this feature may be identified with the Si I UV 1 multiplet, consisting of six lines with wavelengths between 2506 and $2528 \AA$. Below we will show that in iron-rich material, in which Si may reside, the fractional ionization at the present epoch is low and about $70 \%$ of the metals are in a neutral state. Therefore the presence of strong resonance $\mathrm{Si}$ I lines is not surprising. Other Si I UV multiplets lie in the short wavelength region beyond the observed range. Resonance lines of $\mathrm{Si}$ II also lie in this unobserved UV band.

\section{INTERPRETATION OF THE HEAVY-ELEMENT EMISSION}

We begin by reviewing the physical mechanisms involved in the formation of the spectrum of SN 1987A at the late nebular epoch. Fransson \& Kozma (1993) found that the bolometric luminosity at times $t<4 \mathrm{yr}$ is consistent with the absence of a significant contribution from energy sources other than the radioactive nuclides. Here we adopt the commonly used amounts of ${ }^{56} \mathrm{Ni},{ }^{57} \mathrm{Ni}$, and ${ }^{44} \mathrm{Ti}$, namely $0.075 M_{\odot}, 0.0027 M_{\odot}$, and $10^{-4} M_{\odot}$, respectively (Woosley, Pinto, \& Hartmann 1989; Thielemann, Hashimoto, \& Nomoto 1990; Li, McCray, \& Sunyaev 1993). There are direct observational measures of the abundances of ${ }^{56} \mathrm{Ni}$ (Arnett et al. 1989) and ${ }^{57} \mathrm{Ni}$ (Kurfess et al. 1992; Clayton et al. 1992), but the abundance of ${ }^{44} \mathrm{Ti}$ has not been directly measured. In a recent theoretical study, Timmes et al. (1996) find that producing more than $10^{-4} M_{\odot}$ of ${ }^{44} \mathrm{Ti}$ is difficult in core collapse models, but making much less is easy. For the likely progenitor mass of SN $1987 \mathrm{~A}, a^{44} \mathrm{Ti}$ mass $\lesssim 0.6 \times 10^{-4} M_{\odot}$ is indicated. The observational study by Wang et al. (1996) indicates a ${ }^{44} \mathrm{Ti}$ mass as high as $4 \times 10^{-4} M_{\odot}$. In this paper we use a ${ }^{44} \mathrm{Ti}$ mass of $10^{-4} M_{\odot}$ in much of the discussion, but we also derive an approximate ${ }^{44} \mathrm{Ti}$ mass from the [O $\left.\mathrm{I}\right]$ line strength. The radioactive lifetimes for ${ }^{56} \mathrm{Co}$ and ${ }^{57} \mathrm{Co}$ are 111.26 days and $1.07 \mathrm{yr}$, respectively. The lifetime of ${ }^{44} \mathrm{Ti}$ is more uncertain; a recent estimate by Meissner et al. (1995) is $58 \pm 10 \mathrm{yr}$. Because of the small age for SN 1987A, the uncertainty does not affect our results. The average energy per decay is $3.61 \mathrm{MeV}$ in $\gamma$-rays (annihilation of positrons is included) and $0.12 \mathrm{MeV}$ in positrons for ${ }^{56} \mathrm{Co}, 0.125 \mathrm{MeV}$ in $\gamma$-rays for ${ }^{57} \mathrm{Co}$, and $2.13 \mathrm{MeV}$ in $\gamma$-rays (annihilation radiation included) and $0.597 \mathrm{MeV}$ in positrons for ${ }^{44} \mathrm{Ti}$ (Browne, Firestone, \& Shirley 1986).

The energy radiated now by SN 1987A is expected to be composed of three physically distinct components: (1) far infrared thermal emission of cool gas with a temperature on the order of $10^{2} \mathrm{~K}$, (2) UV/optical radiation produced by the instantaneous reprocessing of the radioactive decay energy through collisions with fast electrons, and (3) recombination emission of hydrogen ionized at a previous epoch. Only the last two components contribute to the HST spectrum at the present epoch. Here we discuss the second component, which involves the formation of metal lines. 
Among the radioactive nuclides, ${ }^{44} \mathrm{Ti}$ is the only important power source at the present epoch. The luminosity of $10^{-4} M_{\odot}$ of ${ }^{44} \mathrm{Ti}$ at $t=7.87 \mathrm{yr}$ is $10^{36} \mathrm{ergs} \mathrm{s}^{-1}$ in the kinetic energy of positrons and $4 \times 10^{36} \mathrm{ergs} \mathrm{s}^{-1}$ in $\gamma$-rays. Because of the small optical depth for $\gamma$-rays, positrons dominate the power input. In order to illustrate this statement, let us consider a simplified model of SN 1987A: a homogeneous, freely expanding $(v=r / t)$ envelope with mass $14 M_{\odot}$ and kinetic energy $1 \times 10^{51}$ ergs (see Shigeyama \& Nomoto 1990). For this model the outer velocity is $v_{0}=3450 \mathrm{~km} \mathrm{~s}^{-1}$ and the density $\rho \approx 1.3 \times 10^{-17} \mathrm{~g} \mathrm{~cm}^{-3}$ at $t=7.87 \mathrm{yr}$. The optical depth of the envelope to $\gamma$-rays is $\tau_{\gamma}=k_{\gamma} \rho v_{0}$ $t \approx 0.03$ (where $k_{\gamma}=0.03 \mathrm{~cm}^{2} \mathrm{~g}^{-1}$ is the absorption coefficient for $\sim 1 \mathrm{MeV} \gamma$-rays; Colgate, Petschek, \& Kriese 1980). This means that the present-day supernova luminosity supplied by $\gamma$-rays from ${ }^{44} \mathrm{Ti}$ is $\sim 10^{35} \mathrm{ergs} \mathrm{s}^{-1}$. Positrons with an energy of $0.6 \mathrm{MeV}$ have a range of $0.3 \mathrm{~g} \mathrm{~cm}^{-2}$ (e.g., Longair 1992, p. 85), which is smaller than the radial column density of the envelope $\rho v_{0} t \approx 1 \mathrm{~g} \mathrm{~cm}^{-2}$. Therefore, practically all the positron energy is deposited in the envelope. The presence of a weak magnetic field can easily increase the efficiency of deposition to $100 \%$. These considerations demonstrate that at the present epoch ${ }^{44} \mathrm{Ti}$ positrons deposit 10 times more power in the envelope than $\gamma$-rays do.

This analysis has an important implication: with the exception of $H \alpha$, the UV/optical spectrum of the present day SN 1987A must be dominated by the line-emitting region coincident with the region of positron-energy deposition. Depending on the strength of the magnetic field and the degree of mixing, this region may be bounded by ironrich material or may also include oxygen-rich and possibly even hydrogen-rich material. The line luminosities and their evolution permit us to put some constraints on the fraction of positrons escaping from the iron-rich material into regions of different composition. The primary mechanisms for the creation of photons in the case of instant energy reprocessing are nonthermal excitation and ionization (with subsequent recombination) by fast electrons and degradation of UV photons into low energy photons by scattering in the optically thick resonance metal lines in the metal-rich core and in the hydrogen envelope.

\subsection{UV Magnesium Emission Feature}

The $2825 \AA$ emission feature is presumably formed by a combination of emission and scattering in the $\mathrm{Mg}$ II $\lambda \lambda 2795$, 2803 and $\mathrm{Mg}$ I $\lambda 2852$ resonance lines (Wang et al. 1996). With this identification, we use a simple model of line formation to investigate the density of the scattering envelope in the outer layers.

We assume that the distribution of the net emissivity in both lines is $j=j_{0}=$ const for $v \leq v_{e}=2000 \mathrm{~km} \mathrm{~s}^{-1}$ and $j=j_{0}\left(v / v_{e}\right)^{-4}$ for $v>v_{e}$, where the velocity parameters are chosen to provide a good fit to the $\mathrm{Mg}$ I] $\lambda 4571$ line. The scattering halo is characterized by the distribution of the Sobolev optical depth with velocity in the outer region of the envelope: $\tau=\left(v / v_{1}\right)^{-k}$, where we use $k=8$, which describes the density distribution in the region $4000-10,000$ $\mathrm{km} \mathrm{s}^{-1}$ (Shigeyama \& Nomoto 1990), and $v_{1}$ is the velocity at optical depth 1 . The detailed distribution of $\tau$ in the optically thick region is irrelevant for the line profile because the scattering is strongly conservative. The thermalization parameter in this line is given by the product of the ratio of the collisional deexcitation rate to the spontaneous emission rate, $\epsilon_{21}=q_{21} n_{e} / A_{21}$, with the Sobolev optical depth, $\tau_{12}$. At the present epoch, the thermalization parameter is negligibly small $\left(\epsilon_{21} \tau_{12} \sim 10^{-8}\right)$. Therefore the source function for this line is equal to the local average intensity of the incident radiation.

The assumption that the radial dependence of the optical depth in the $\mathrm{Mg}$ II resonance line is similar to the density profile is valid because almost all the $\mathrm{Mg}$ is in a singly ionized state. The radial dependence of the fractional ionization of $\mathrm{Mg}$ I can cause a deviation from the assumed similar $\left(v^{-8}\right)$ radial dependence of the $\mathrm{Mg}$ I density. However, because of the steep radial dependence of the density, the overall shape of the $\mathrm{Mg}$ I profile will be determined primarily by the parameter $v_{1}$, which generally is different from the corresponding value for $\mathrm{Mg}$ II and must be determined from a comparison with the observations.

The radiative coupling between $\mathrm{Mg}$ I and $\mathrm{Mg}$ II lines due to the Doppler effect was treated according to the recipe of Rybicki \& Hummer (1978). We did not take into account the scattering of the hydrogen two-photon continuum, which has only a minor effect (less than $5 \%$ ) on line formation. However, the level of the background continuum was chosen at the level of the two-photon continuum in the region of $2800 \AA$. The dust core was modeled by a homogeneous sphere with the radius determined by $v_{d}=1800 \mathrm{~km}$ $\mathrm{s}^{-1}$ and optical depth $\tau_{d}=1$, which are close to the parameters found by Lucy et al. (1991). We have found that this model is consistent with the $\mathrm{H} \alpha$ profile.

The best fit using this model (Fig. 6a) is far from perfect. Although the red half of the profile corresponding to the Mg I $\lambda 2852$ line fits well, the narrow net emission of $\mathrm{Mg}$ II $\lambda 2800$ is in apparent disagreement with the wide blue part of the observed profile. In an attempt to resolve the problem, we noticed that the region affected by the $\mathrm{Mg}$ II $\lambda 2800$ absorption may contain emission lines of Fe II UV 62 and UV 63. These multiplets have the largest branching ratios among multiplets with ${ }^{4} D^{o}$ and ${ }^{4} F^{o}$ upper states. Another two subordinate multiplets of lower intensity, UV 35 and UV 36, fall in the range $2350-2390 \AA$ and therefore must be reprocessed by the resonance multiplets UV 3 and UV 2. The presence of strong emission of Fe II UV 62 and UV 63 cannot be ruled out. In order to check this possibility, we took the 12 strongest lines of the UV 62 and UV $63 \mathrm{Fe}$ II multiplets to have fluxes proportional to $g_{u} A_{u l}$. The resultant synthetic spectrum (Fig. $6 b$ ) shows good agreement with the observations in the region $2700-2800 \AA$. This particular model suggests that the contribution of the $\mathrm{Fe}$ II UV 62 and 63 emission lines is $50 \%$ of the flux in this feature, $30 \%$ of the flux comes from $\mathrm{Mg}$ II $\lambda 2800$, and $20 \%$ of the flux comes from $\mathrm{Mg}$ I 22852 , the luminosity of the latter line being equal to that of the $\mathrm{Mg} \mathrm{I}] 24571$ line. In the second model (Fig. 6b), the velocity at the level of Sobolev optical depth $\tau=1$ is $6200 \pm 300 \mathrm{~km} \mathrm{~s}^{-1}$ for $\mathrm{Mg}$ I $\lambda 2852$ and $9000 \pm 500 \mathrm{~km} \mathrm{~s}^{-1}$ for the $\mathrm{Mg}$ II $\lambda 2800$ line.

Wang et al. (1996) suggested that the large radius of SN 1987A in the near-UV wavelength region (filter F275W) compared to its radius in the optical wavelength region (filter F501N) in the HST image in January 1994 (Jakobsen et al. 1994) arises from the contribution of the $\mathrm{Mg}$ II resonance line to the UV wavelength region. One can identify the velocity at the radius $\tau=1$ in the $\mathrm{Mg}$ II line, $v_{1}=9000$ $\mathrm{km} \mathrm{s}^{-1}$, with the outer edge of the ejecta in the near UV. The reason is the abrupt increase in the rate of decline of the intensity in the $\mathrm{Mg}$ II line, from $I \propto v^{-2}$ for $v<v_{1}$ to 


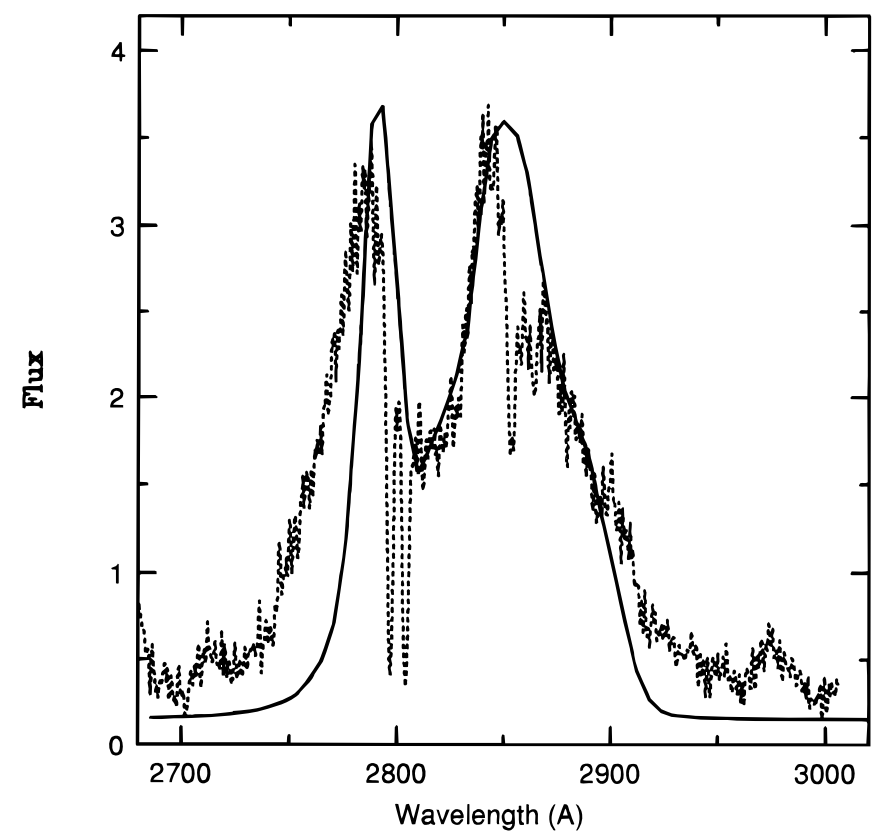

FIG. $6 a$

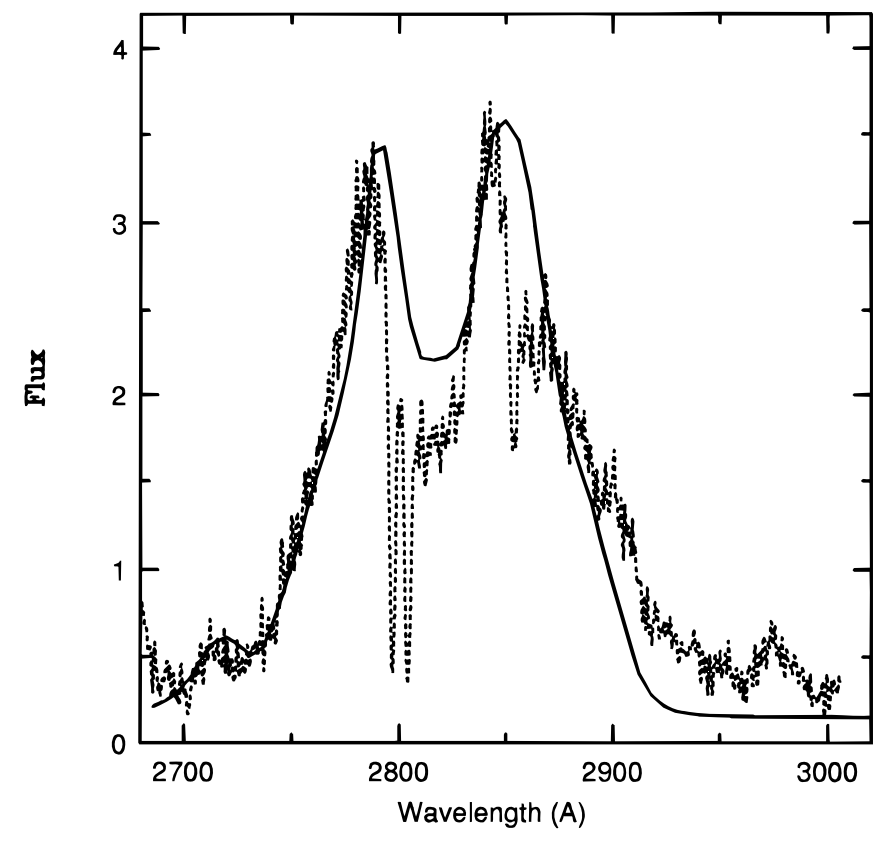

FIG. $6 b$

FIG. 6. - The emission feature at $2825 \AA$ at $t=7.87 \mathrm{yr}$ (dotted lines) and two versions of the synthetic spectrum (solid lines): (a) only Mg II $\lambda 2800$ and Mg I $\lambda 2852$ emission present, $(b)$ in addition to $\mathrm{Mg}$ II and $\mathrm{Mg}$ I, lines of the Fe II multiplets UV 62 and UV 63 contribute half of the total flux.

$I \propto v^{-k-2} \approx v^{-10}$ for $v>v_{1}$, which results in a steep drop in the brightness of the $\mathrm{Mg}$ II line at an impact parameter $v>v_{1}$. The velocity $v_{1}$ in the $\mathrm{Mg}$ II resonance line is expected to decrease with time as $v_{1} \propto t^{-2 / k}$. For $k=8$, the present epoch velocity of $9000 \mathrm{~km} \mathrm{~s}^{-1}$ corresponds to the velocity $9300 \mathrm{~km} \mathrm{~s}^{-1}$ at the epoch of HST imaging ( $t=2522$ days), which agrees fairly well with the $H S T$ result, namely $9400 \mathrm{~km} \mathrm{~s}^{-1}$ for a distance of $50 \mathrm{kpc}$ (Jakobsen et al. 1994).

The velocities inferred at the level $\tau=1$ for the $\mathrm{Mg}$ I and Mg II UV lines can be used to find the number densities of $\mathrm{Mg}$ II and $\mathrm{Mg}$ I at the corresponding radii. Using the oscillator strengths from Kurucz \& Peytremann (1975), we find $n(\mathrm{Mg}$ II $)=5.8 \times 10^{-3} \mathrm{~cm}^{-3}$ at $v=9000 \mathrm{~km} \mathrm{~s}^{-1}$ and $n(\mathrm{Mg} \mathrm{I})=2.9 \times 10^{-3} \mathrm{~cm}^{-3}$ at $v=6200 \mathrm{~km} \mathrm{~s}^{-1}$. Extrapolating the density of $\mathrm{Mg}$ II inward according to the law $n \propto v^{-8}$, we obtain $n(\mathrm{Mg} \mathrm{II})=0.11 \mathrm{~cm}^{-3}$ at $v=6200 \mathrm{~km}$ $\mathrm{s}^{-1}$, which gives the ratio $n(\mathrm{Mg} \mathrm{I}) / n(\mathrm{Mg} \mathrm{II})=0.03$, with a possible error of a factor of 2 . Because the expected relative density of $\mathrm{Mg}$ III is negligibly small, the density of $\mathrm{Mg}$ II is equal to the total density of $\mathrm{Mg}$. With the assumption of a metal abundance one-third of solar (Rosa \& Mathis 1987), the density of $\mathrm{Mg}$ at the level $9000 \mathrm{~km} \mathrm{~s}^{-1}$ obtained above results in a total matter density at this radius of $\rho=1.45 \times 10^{-21} \mathrm{~g} \mathrm{~cm}^{-3}$, with an error of $0.3 \mathrm{dex}$.

The hydrodynamic model 14E1 of Shigeyama \& Nomoto (1990) $\left(M=14.6 M_{\odot}, E=10^{51}\right.$ ergs $)$ predicts a density of $\rho=1.67 \times 10^{-21} \mathrm{~g} \mathrm{~cm}^{-3}$ at the velocity $9000 \mathrm{~km} \mathrm{~s}^{-1}$ $(t=7.87 \mathrm{yr})$, which agrees with the value found above. On the other hand, the model 11E0.6 from the same paper predicts a density $\rho=0.67 \times 10^{-21} \mathrm{~g} \mathrm{~cm}^{-3}$, which is a factor of 2 lower than our estimate but is probably still acceptable.

\subsection{Energy and Ionization Balance in Iron-rich Material}

The strong Fe II emission lines of UV 1 and UV 2 probably originate from a combination of two processes: net emission of the iron-rich material and scattering of the resulting photons in different lines of these multiplets in the iron-rich material and in the hydrogen-rich envelope. The effect of scattering for iron is more complex than for the magnesium blend because of the line interlocking in multiplets (i.e., line coupling via a common upper level). We consider here only the line-emitting region coinciding with the iron-rich inner zone of the envelope. All the iron is assumed to be the result of ${ }^{56} \mathrm{Ni}$ decay and to lie in the region $v<1700 \mathrm{~km} \mathrm{~s}^{-1}$. The expected density of $\mathrm{Fe}\left(M_{\mathrm{Fe}}=0.075\right.$ $M_{\odot}$; Arnett et al. 1989) at $t=7.87 \mathrm{yr}$ is then $n=5 \times 10^{3} / \mathrm{f}$ $\mathrm{cm}^{-3}$, where the filling factor of iron peak elements is likely to be $f \approx 0.3-0.5$ (Li et al. 1993). Adopting $f=0.5$, we find $n(\mathrm{Fe})=10^{4} \mathrm{~cm}^{-3}$, with a volume $V=1.58 \times 10^{50} \mathrm{~cm}^{3}$ occupied by the iron. The ionization state of $\mathrm{Fe}$ may be found from the ionization balance (the steady-state approximation for iron is still valid) assuming that positrons from ${ }^{44} \mathrm{Ti}$ decay are the major source of energy and that all the positrons deposit their energy in the iron. The corresponding ionization equation for the iron ionized fraction $x=n(\mathrm{Fe} \mathrm{II}) / n(\mathrm{Fe})$ is

$$
\alpha x^{2} n^{2} V=0.5(1-x)\left(1-x^{0.2}\right) \chi^{-1} L_{\beta},
$$

where $\alpha$ is the recombination coefficient to $\mathrm{Fe}$ I (taken from Woods, Shull, \& Sarazin 1981), $n$ is the total particle number of $\mathrm{Fe}$ (initially the particle number of ${ }^{56} \mathrm{Ni}$ ), $L_{\beta}=$ $10^{36}$ ergs s $^{-1}$ is the total luminosity released as kinetic energy of positrons in beta decays of $10^{-4} M_{\odot}$ of ${ }^{44} \mathrm{Ti}$, and $\chi$ is the ionization potential of $\mathrm{Fe}$ I. The factor of $\left(1-x^{0.2}\right)$ on the right-hand side describes the fraction of the energy going into ionization and into excitation (Kozma \& Fransson 1992). The energy is shared approximately equally between these two channels, which accounts for the factor of 0.5 on the right-hand side of equation (1).

Because the recombination coefficient depends on the electron temperature, we simultaneously solved equation (1) with the thermal balance equation with the $26 \mu \mathrm{m}$ Fe II line 
as the major cooling agent by

$$
\Lambda(26 \mu \mathrm{m}) x^{2} n^{2} V=x^{0.2} L_{\beta},
$$

where $\Lambda$ is the cooling function given by

$$
\Lambda(26 \mu \mathrm{m})=\frac{q_{12} E_{12}}{1+n_{e} q_{21} / A_{21}} .
$$

Here, $q_{12}$ and $q_{21}$ are the collisional excitation and deexcitation rates, $E_{12}=7.6 \times 10^{-14}$ ergs is the energy of the transition, and $A_{21}=0.00213 \mathrm{~s}^{-1}$ (Nussbaumer \& Storey 1980 ) is the spontaneous emission rate. The collision rates depend on the collision strength, which we take to be 7, based on an extrapolation of the results of Pradhan \& Zhang (1993). At $t=7.87 \mathrm{yr}$, the solution of equations (1) and (2) yields $x=0.27$ for the ionization of iron and an electron temperature $T=160 \mathrm{~K}$. For these parameters, $\alpha n_{e} t \approx 3.7$, which means that the steady-state approximation is a good first approximation. If iron peak elements and silicon (a product of oxygen burning) are mixed, then for a mass of silicon comparable to that of iron (Thielemann et al. 1990), the positron energy will be shared roughly equally between iron and silicon. In that case the power deposited into iron decreases by a factor of 2 , the density increases by a factor of 2 , and therefore the ionized fraction decreases roughly by a factor $2^{3 / 2}$, becoming $x \approx 0.1$.

The low ionization of iron suggests that both Fe II and $\mathrm{Fe}$ I emission lines should be present in the UV/optical spectrum. A similar conclusion applies to $\mathrm{Si} \mathrm{I}$ and $\mathrm{Si}$ in lines. The possible contribution of $\mathrm{Fe}$ I and $\mathrm{Si}$ I emission lines suggested above thus finds support in the dominant abundance of neutral metals in the iron-rich zone.

We have assumed that the Fe II $26 \mu \mathrm{m}$ line dominates the radiative loss rate. Another possible contributor to the radiative losses is the $\mathrm{Fe}$ I $24 \mu \mathrm{m}$ line, which, neglecting collisional deexcitation, has a luminosity given by

$$
\frac{L(\mathrm{Fe} \mathrm{I})}{L(\mathrm{Fe} \mathrm{II})} \approx \frac{10}{9} \frac{\Omega(\mathrm{Fe} \mathrm{I})}{\Omega(\mathrm{Fe} \mathrm{II})} \frac{(1-x)}{x},
$$

where $\mathrm{Fe}$ I denotes the $24 \mu \mathrm{m}$ line, $\mathrm{Fe}$ II denotes the $26 \mu \mathrm{m}$ line, and $\Omega$ is the collision strength. The collision strength of the $24 \mu \mathrm{m}$ line has been poorly known, but Pelan \& Berrington (1997) recently gave a value of 0.03 at $T \approx 200 \mathrm{~K}$. When this value is substituted into the above equation with $\Omega(\mathrm{Fe}$ II $)=7$ and $x=0.27$, we find $L(\mathrm{Fe} \mathrm{I}) \approx 10^{-2} L(\mathrm{Fe}$ II $)$ and the $\mathrm{Fe} \mathrm{I}$ line can be neglected.

Each of the $\mathrm{Fe}$ II resonance transitions 1-3 (with the exception of the Fe II $\lambda 2382$ line of the UV 2 multiplet) has a common upper level 3 with some subordinate transition 3-2. In this situation, the excitation of level 3 results in the escape of photons in the UV line with the effective branching ratio

$$
p_{\mathrm{UV}}=A_{31} \beta_{13} /\left(A_{31} \beta_{13}+A_{32} \beta_{12}\right),
$$

where $A_{k i}$ is the Einstein coefficient and $\beta_{i k}$ is the Sobolev escape probability. At the present epoch the optical depth in the subordinate lines is $\ll 1$ but that in the resonance lines is large, so the branching ratio for subordinate lines may be high. We estimate the effective branching ratio for the UV $\mathrm{Fe}$ II multiplets by assuming that the rate of excitation of the upper level is proportional to its statistical weight. Summing up the branching ratios of all the UV lines with the proper weights, assuming the Boltzmann population of ground-term fine-structure levels for $T=160 \mathrm{~K}$ (the electron density is of the order of the critical density), we find effective branching ratios of $0.64,0.51$, and 0.18 for UV 1 , UV 2, and UV 3, respectively. These numbers may increase by $10 \%-20 \%$ if one takes into account that the finestructure levels are not collisionally saturated. These values of the branching ratios show a high probability for photons to escape in Fe II UV resonance lines.

The major parameters determining the branching ratio of the $\mathrm{Fe}$ II UV resonance lines are the $\mathrm{Fe}$ II density and the temperature, the latter being particularly important at the present epoch because the optical depths of the groundlevel resonance lines are still very high $\left(\sim 10^{5}\right)$. The low temperature reduces the optical depths of the resonance lines from excited fine-structure levels, thus decreasing the effective branching ratio for subordinate lines. This trend is demonstrated observationally by the dramatic decrease by a factor of $\sim 4$ of the intensity ratio (Fe II $\lambda 5018) /(\mathrm{Fe}$ II UV 2) recorded in the HST spectra between $5.1 \mathrm{yr}$ (Wang et al. 1996) and the present.

In the case of multiplets UV 1 and UV 2, the strongest subordinate multiplets (multiplets 1 and UV 60, respectively) lie in the near-UV region and are affected by reprocessing in the resonance lines of neutral metals, particularly $\mathrm{Fe}$. The fate of UV 60 radiation is of particular interest. The estimated branching ratio for multiplet UV 60 $(p \approx 0.49)$ suggests that in the range of $2900-3000 \AA$ one expects the presence of an emission feature with a total observed flux of $2 \times 10^{-14} \mathrm{ergs} \mathrm{s}^{-1} \mathrm{~cm}^{-2}$, which exceeds the observed flux in this region by a factor of 4 . The conspicuous absence of UV 60 may be explained by the reprocessing of this emission by UV metal lines, particularly in the Fe I UV 1 multiplet, which has transitions in the range 2912-2973 $\AA$. The radiation absorbed by Fe I UV 1 should be reemitted in the subordinate lines of multiplet 21 in the wavelength region $3728-3799 \AA$, thus contributing to the $3730 \AA$ feature. This reasoning provides some justification for suggesting the presence of $\mathrm{Fe} I$ lines of multiplet 21 in the 3730 A emission $(\S 3)$.

The dominance of singly ionized iron in the hydrogen envelope may result in an additional increase in the intensity of the resonance Fe II multiplets through UV metal-line radiation scattering in these optically thick resonance lines. Given the intensity of the UV pseudo-continuum, this process could contribute $10 \%-20 \%$ of the total intensity of the Fe II UV 1 and UV 2 multiplets.

\subsection{UV/optical Fe Lines}

The low ionization of iron in the iron-rich material predicted by the radioactive model suggests that a large fraction of the energy from ${ }^{44} \mathrm{Ti}$ positrons is deposited into neutral material. We now discuss the expected UV/optical luminosity of neutral and singly ionized species from the iron-rich gas.

The fraction of the positron energy going into heating is $h \approx x^{0.2}$, which is radiated in the far-IR band, particularly in the $26 \mu \mathrm{m}$ line. The remaining energy fraction, $1-h$, goes into ionization and excitation with branching ratios $g$ and $1-g$, respectively. UV and optical photons are also produced by recombination, which must be included in the computation of the energy flow. Ion excitation must also include ionization with simultaneous excitation (the process $e+\mathrm{X} \rightarrow \mathrm{X}^{+*}+2 e$ ), which has a branching ratio $y$. In the absence of relevant cross section data, we use the same value of $y$ for both processes with $Z=0 \rightarrow 1$ and those with $Z=1 \rightarrow 2$. 
The branching ratio of the energy flow to neutral atomic lines in the steady state is then

$$
p_{0}=(1-x)[1-g+g(1-y)],
$$

where the second term in brackets corresponds to recombinations corrected for energy loss to excitation of ions with $Z=1$, while the other terms describe the collisional excitation of neutrals. The branching ratio of the energy flow to the lines of ions with $Z=1$ is

$$
p_{1}=x[1-g+g(1-y)]+g y(1-x),
$$

where the first term is similar to the right-hand side of equation (4) and the second term corresponds to excitation followed by ionization. The fractions of the ${ }^{44} \mathrm{Ti}$ positron luminosity that are radiated in the UV/optical wavelength region are $(1-h) h p_{0}$ and $(1-h) p_{1}$ for neutral and ionized species, respectively; a fraction $h$ is emitted in the far-IR wavelength region. In our estimates below, we use an ionized fraction $x=0.27$ for unmixed iron as obtained above and adopt the approximation that the energy going into ionization is practically equal to the energy going into excitation, i.e., $g=0.5$.

Because of the uncertainty in the branching ratio of the excitation of ions during collisional ionization, we consider three cases: $y=0$ (process $e+\mathrm{X} \rightarrow \mathrm{X}^{+*}+2 e$ prohibited), $y=0.5$, and $y=0.8$. We expect $y \sim 0.5$; as an example, we note the well-studied case of $\mathrm{O}$ I ionization by electron impact, for which $66 \%$ of ionizations at energies more than $100 \mathrm{eV}$ leave $\mathrm{O}$ II in the excited state (see Laher \& Gilmore 1990).

The resulting fractions for the energy flow into different channels are given in Table 3. They demonstrate that (1) the bulk $(77 \%)$ of the thermal energy $(T \approx 160 \mathrm{~K})$ is emitted in far-IR lines; (2) in the plausible case $0.5 \leq y<0.8$, the $\mathrm{UV} /$ optical emission is shared roughly equally between neutral and singly ionized atoms with some predominance of emission from neutrals; (3) the predicted UV/optical luminosity of $\mathrm{Fe}$ II is of the order of $10 \%$ of the positron luminosity of ${ }^{44} \mathrm{Ti}$, i.e., $\sim 10^{35} \mathrm{ergs} \mathrm{s}^{-1}$ for $10^{-4} M_{\odot}$ of ${ }^{44} \mathrm{Ti}$. These results are not sensitive to a moderate variation of ionized fraction $x$ and ionization branching ratio $g$.

At $t=7.87 \mathrm{yr}$, the observed luminosity in Fe II UV 1 and UV 2 was $3.4 \times 10^{34} \mathrm{ergs} \mathrm{s}^{-1}$. If we add the luminosity of optical lines identified with $\mathrm{Fe}$ II and a suggested contribution of $1.4 \times 10^{34}$ ergs s$^{-1}$ in the $2825 \AA$ magnesium line, then the total Fe II luminosity increases to $8.1 \times 10^{34}$ ergs $\mathrm{s}^{-1}$. In fact, the internal dust absorption increases this estimate by a factor depending on the dust optical depth and the details of the distribution of the iron-rich material relative to dusty matter. With the effective optical depth of the dusty zone $\tau_{d}=1$, the calculated correction factor in the spherical homogeneous model is 1.5 , which means that the total luminosity of $\mathrm{Fe}$ II lines in the observed spectrum may be as high as $1.2 \times 10^{35}$ ergs $\mathrm{s}^{-1}$. Forbidden Fe II lines in

TABLE 3

ENERGY BALANCE OF IRON-RICH GAS

\begin{tabular}{cccc}
\hline \hline & \multicolumn{3}{c}{ Percent Contribution } \\
\cline { 2 - 4 } ENERgy Channels & $y=0$ & $y=0.5$ & $y=0.8$ \\
\hline Far-IR lines ..................... & 77 & 77 & 77 \\
UV/optical lines (neutrals) $\ldots \ldots \ldots \ldots$ & 17 & 13 & 10 \\
UV/optical lines (ions, $Z=1) \ldots \ldots$ & 6 & 9 & 10 \\
\hline
\end{tabular}

the near-IR wavelength region do not contribute noticeably because the major radiative cascade to the ground level goes through optical forbidden lines and far-IR lines of the ground term.

According to our estimates, the luminosity of the $\mathrm{UV} /$ optical Fe II lines is $10 \%$ of the deposited luminosity, which then should be $\sim 1.2 \times 10^{36}$ ergs. This requires that all the positron luminosity from $\sim 1.2 \times 10^{-4} M_{\odot}$ of ${ }^{44} \mathrm{Ti}$ must be deposited in the iron. Bearing in mind errors in the observational flux and uncertainties in the mass of ${ }^{44} \mathrm{Ti}$, we do not rule out the possibility that some fraction of the positron energy could be deposited to other species, e.g., to silicon.

The model prediction of a large UV/optical luminosity of iron-rich core lines of neutral species $\left(\sim 10^{35}\right.$ ergs $\left.\mathrm{s}^{-1}\right)$ raises the question of where these lines are. The proposed identification of $\mathrm{Fe}$ I $\lambda 3730$ and $\mathrm{Si}$ I $\lambda 2514$ covers only about $20 \%$ of the expected amount. One possible solution is that some fraction of the luminosity of neutral atoms (Fe I and Si I) lies in the region $\lambda<2000 \AA$. As a result of UV photon degradation, a significant fraction of the UV emission may be converted into numerous optical and near-IR permitted and forbidden lines. These lines should contribute to the observed quasi-continuum with a total observed luminosity $\approx 7.3 \times 10^{34} \mathrm{ergs} \mathrm{s}^{-1}$. Among the strongest lines of neutral atoms from iron-rich material must be forbidden $\mathrm{Si}$ I lines of multiplet ${ }^{3} P-{ }^{1} D(\lambda \approx 1.6 \mu \mathrm{m})$ and the Fe I multiplet $a^{5} D-a^{5} F(\lambda \approx 1.4 \mu \mathrm{m})$. Both transitions cover a large fraction of the population flow down to the ground level. In our opinion, this mechanism is responsible for the recently detected strong emission in the near-infrared [Fe I] and [Si I] lines from SN 1987A (Spyromilio \& Leibundgut 1995). Some Fe I emission may also contribute to the Fe II UV 1 and UV 2 bands by scattering in the resonance lines of Fe II.

The high efficiency for absorption of the ${ }^{44} \mathrm{Ti}$ positron energy in the iron-rich gas suggested by the high luminosity in the Fe II lines has an interesting implication. With an initial energy of $0.6 \mathrm{MeV}$, the positron range is $0.3 \mathrm{~g} \mathrm{~cm}^{-2}$. At the present epoch, with a density of iron-rich material $\sim 10^{-18} \mathrm{~g} \mathrm{~cm}^{-3}$, this range corresponds to a velocity range $\sim 1.2 \times 10^{4} \mathrm{~km} \mathrm{~s}^{-1}$, which is seven times larger than the characteristic velocity of the iron-rich core. In this case, positrons must deposit their energy in the more abundant neighboring elements like $\mathrm{O}, \mathrm{He}$, and $\mathrm{H}$, unless the magnetic field prevents positrons from escaping the iron-rich material. A lower limit on the magnetic field strength may be estimated from the condition that the gyroradius $r_{\mathbf{B}}=$ $p c / e B$ (where $p$ is the positron momentum) should be significantly less than the characteristic size of the region occupied by iron-rich material. Assuming that the gyroradius is $r_{\mathrm{B}}<$ $0.1\left(v_{\mathrm{Fe}} t\right)$, we find a lower limit to the magnetic field strength in the present-day envelope of $B>5 \times 10^{-13} \mathrm{G}$. The reasonable assumption of magnetic flux conservation during the expansion imposes a lower limit on the initial magnetic field strength in the presupernova silicon mantle. Taking the radius of the silicon mantle $\sim 10^{9} \mathrm{~cm}$, we obtain an initial field of $B_{0}>10^{3} \mathrm{G}$. This value is reasonable in that it allows the magnetic pressure to be significantly less than the material pressure.

\subsection{Oxygen-Line Excitation}

At late epochs $(t>1000$ days), when the temperature in the oxygen material falls below $1000 \mathrm{~K}$, the $[\mathrm{O} \mathrm{I}] \lambda 6300$ line 
is excited by fast electrons (Fransson \& Kozma 1993). The integrated effect of the nonthermal excitation of this line can be conveniently expressed through the number of photons, $\psi$, emitted per ionization. The luminosity of the $[\mathrm{O} I] \lambda 6300$ doublet is related to the total rate of oxygen ionization according to

$$
L(\lambda 6300)=\psi L_{d} \frac{E_{\mathrm{ex}}}{\chi_{\mathrm{eff}}},
$$

where $L_{d}$ is the total deposited power into the oxygen material, $E_{\text {ex }}$ is the excitation potential of the ${ }^{1} D$ level (1.96 $\mathrm{eV}$ ), and $\chi_{\text {eff }}$ is the effective ionization potential of $\mathrm{O} \mathrm{I}$, which for pure oxygen material depends on the ionized fraction $(x)$ approximately as $\chi_{\text {eff }}=21.6 /\left(1-x^{0.4}\right) \mathrm{eV}$ (Kozma \& Fransson 1992). Assuming that oxygen and ${ }^{44} \mathrm{Ti}$ are macroscopically and homogeneously mixed in a sphere with boundary velocity $v_{b}=1700 \mathrm{~km} \mathrm{~s}^{-1}$, we obtain, in the limit of small optical depth for $\gamma$-rays $\left(\tau_{\gamma}<1\right)$, that the power deposited in the oxygen material in terms of the $\gamma$-ray luminosity $L_{\gamma}$ of ${ }^{44} \mathrm{Ti}$ decays as

$$
L_{d} \approx \frac{3 k_{\gamma} M_{O} L_{\gamma}}{4 \pi\left(v_{b} t\right)^{2}}
$$

where $k_{\gamma}=0.03 \mathrm{~cm}^{2} \mathrm{~g}^{-1}$ is the absorption coefficient for $\gamma$-rays and $M_{O}$ is the oxygen mass. We assume that the positrons do not penetrate the oxygen.

The $\psi$ value can be calculated provided the steady-state energy spectrum of the secondary electrons is known. We found this spectrum for pure oxygen in the energy range $E<200 \mathrm{eV}$ by numerically solving the kinetic equation for the distribution function of secondary electrons. Our procedure was similar to that of Kozma \& Fransson (1992). The following simplifications were used: only electron collisions with neutral oxygen and Coulomb losses were considered and excitation of the ${ }^{1} D$ term and Coulomb losses were treated in the approximation of continuous loss. The ionization was considered in the approximation of constant loss $\Delta E=\gamma \chi$, where $\chi$ is the ionization potential and $\gamma$ is a factor close to 2 . In the the region $\chi<E<\gamma \chi$ the loss to ionization was taken to be $\Delta E=E-\chi$. Computations showed that $\psi$ is not sensitive to the $\gamma$-value in the expected range $1.5<\gamma<2.5$; in this range the variation of $\psi$ is less than $2 \%$. In order to provide rapid convergence of the solution, we used the approximation of continuous loss for ionization in the energy range $E>50 \mathrm{eV}$. This method permitted us to find an analytic solution for the distribution function $f(\mathrm{E})$ in the high energy range, which was matched with the numerical solution in the range $E<50 \mathrm{eV}$. The corresponding cross sections were taken from Itikawa \& Ichimura (1990), while the energy distribution of secondary electrons generated by fast electrons at $E>200 \mathrm{eV}$ and above was approximated by the expression (see Shull 1979)

$$
Q_{0}(u)=\frac{2}{\pi\left(1+u^{2}\right)},
$$

where $u=E / E_{0}$, and $E_{0}=0.6 \chi$.

We consider first the "standard" model for the oxygen distribution: oxygen mass $1.5 M_{\odot}$, filling factor 0.1 , constant density, and outer velocity $v_{o}=1700 \mathrm{~km} \mathrm{~s}^{-1}$ (see Li \& McCray 1992). At $t=7.87 \mathrm{yr}$, the oxygen number density in this model is $3.6 \times 10^{6} \mathrm{~cm}^{-3}$. The adopted kinetic temperature of the oxygen-rich material is $\sim 50 \mathrm{~K}$, the value obtained by the extrapolation of results from Fransson et al. (1996). For a ${ }^{44} \mathrm{Ti}$ mass of $10^{-4} M_{\odot}$, we found that the $\gamma$-ray power deposited in the oxygen is $4.7 \times 10^{34} \mathrm{ergs} \mathrm{s}^{-1}$ (see eq. [7]), the oxygen ionization fraction $x \approx 5 \times 10^{-4}$ with the electron concentration is $n_{e}=2 \times 10^{3} \mathrm{~cm}^{-3}$, and $\psi=0.9$. According to eq. (6), the luminosity in the [O I] doublet is then $L(\lambda 6300)=4 \times 10^{33} \mathrm{ergs} \mathrm{s}^{-1}$, which is a factor of 1.3 lower than the observed value $\left(5.2 \times 10^{33} \mathrm{ergs} \mathrm{s}^{-1}\right)$. The difference indicates either a higher mass of oxygen $\left(2 M_{\odot}\right)$ or a higher ${ }^{44} \mathrm{Ti}$ mass.

Computations of ionization and $\psi$ for higher ${ }^{44} \mathrm{Ti}$ mass show that the observed luminosity $\left(5 \times 10^{33} \mathrm{ergs} \mathrm{s}^{-1}\right)$ is reproduced for $2 \times 10^{-4} M_{\odot}$ of ${ }^{44} \mathrm{Ti}$. Alternatively, a similar result could be obtained with a ${ }^{44} \mathrm{Ti}$ mass of $10^{-4}$ $M_{\odot}$ but with $5 \%$ of the positron luminosity deposited in the oxygen.

The [O II] $\lambda 3727$ line is presumably caused by the collisional ionization of neutral oxygen with the simultaneous excitation of ${ }^{2} D^{o}$ and ${ }^{2} P^{o}$ terms, which is the mechanism discussed by Wang et al. (1996). According to Laher \& Gilmore (1989), the fraction of the oxygen ionizations going into the excitation of these states is weakly dependent on the electron energy, and at the energy of the maximum cross section $(\approx 150 \mathrm{eV})$, the branching ratios to the ${ }^{2} D^{o}$ and ${ }^{2} P^{o}$ states are $r_{2}=0.27$ and $r_{3}=0.16$, respectively. The [O II] 27324 3-2 transition, with branching ratio $b_{32}=0.8$, will contribute to the excitation of the ${ }^{2} D^{o}$ term, so that the total probability of excitation of this term per oxygen ionization is $p=r_{2}+r_{3} b_{32}=0.4$. These values for the branching ratios yield an intensity ratio for the $[\mathrm{O} \mathrm{II}]$ lines of $I(7320) / I(3727)=0.04$, which precludes identification of the $7300 \AA$ emission line with [O II] $\lambda 7320$ (see Table 2).

The luminosity of the $[\mathrm{O}$ II] $\lambda 3727$ line is then

$$
L(\lambda 3727)=p \frac{E_{\mathrm{ex}}}{\chi_{\mathrm{eff}}} L_{d}\left(1+\frac{n_{e}}{n_{e, \mathrm{cr}}}\right)^{-1},
$$

where the last term in parentheses takes into account collisional deexcitation of the upper level $\left(n_{e, \text { cr }}=1.9 \times 10^{3}\right.$ $\mathrm{cm}^{-3}$ for $\left.T=50 \mathrm{~K}\right)$. Using $n_{e}=2 \times 10^{3} \mathrm{~cm}^{-3}$, as found above, $L_{d}=4.7 \times 10^{34} \mathrm{ergs} \mathrm{s}^{-1}$, and $10^{-4} M_{\odot}$ of ${ }^{44} \mathrm{Ti}$, we find a line luminosity $L(\lambda 3727)=1.6 \times 10^{33} \mathrm{ergs} \mathrm{s}^{-1}$, which is $1 / 7.5$ times the observed value (Table 2 ). With a ${ }^{44} \mathrm{Ti}$ mass of $2 \times 10^{-4} M_{\odot}$ or, alternatively, with $5 \%$ of the positron energy deposited into oxygen material, one obtains $L(\lambda 3727)=2.5 \times 10^{33} \mathrm{ergs} \mathrm{s}^{-1}$, which is only $20 \%$ of the observed luminosity of the $3730 \AA$ feature. This result is generally consistent with the estimates by Wang et al. (1996), who suggested that the [O II] line in the HST spectra at supernova ages of 5 and $6 \mathrm{yr}$ can be explained only by assuming a large ${ }^{44} \mathrm{Ti}$ mass $\left(4 \times 10^{-4} M_{\odot}\right)$ and a large oxygen mass $\left(5-7 M_{\odot}\right)$.

In an attempt to explain the observed [O II] line intensity with a lower mass of oxygen and ${ }^{44} \mathrm{Ti}$, we checked the possibility of a two-component model of the oxygen distribution explaining both the $[\mathrm{O} \mathrm{II}]$ and $\left[\mathrm{O}_{\mathrm{I}}\right]$ lines. Apart from the dense oxygen clouds described by the "standard" model $\left(M=1.5 M_{\odot}, f=0.1\right.$, and $\left.v_{o}=1700 \mathrm{~km} \mathrm{~s}^{-1}\right)$ considered above, we introduced a second, rarefied component that is characterized by a lower oxygen density and a high rate of energy deposition from positrons. The rarefied oxygen could, e.g., be associated with the boundary layer of oxygen clumps. We found that both [O I] and [O II] line luminosities are reproduced at $t=7.87 \mathrm{yr}$ with the follow- 
ing parameters for the rarefied component: oxygen mass of $0.05 M_{\odot}$, filling factor 0.4 , and $30 \%$ of the positron energy deposited into the rarefied component. In this model the dense component, powered by $\gamma$-rays from $2 \times 10^{-4} M_{\odot}$ of ${ }^{44} \mathrm{Ti}$, is responsible primarily for the [O $\left.\mathrm{O}\right]$ emission, while the rarefied component predominantly emits the [O II $]$ line.

However, the two-component model does have a serious drawback. Because of the strong ionization (and hence high $n_{e}$ ) in the rarefied component, the [O II] $\lambda 3727$ emission is subjected to significant collisional deexcitation. Therefore the model predicts a $30 \%$ rise in the [O II] line luminosity over the period 5.1-7.87 yr, which is opposite to the observed drop to half the original value. It may be that the rarefied oxygen component is weak (if it exists at all) and that some other line or lines contribute to the $3730 \AA$ emission region, e.g., $\mathrm{Fe} \mathrm{I}$, as we suggested earlier ( $\$ 3)$.

A secondary result of the oxygen-line analysis is the conclusion that positrons practically do not penetrate into the bulk of the oxygen material: less than $5 \%$ of the positrons can deposit their energy into the bulk of the oxygen. The previous claim (see $\S 4.3$ ) that positrons are trapped in the Fe-rich material by the magnetic field thus finds an independent confirmation. Yet even a small leakage $(5 \%)$ of positrons from $\mathrm{Fe}$-rich to O-rich gas is crucial for the energy balance of oxygen. It is conceivable that the contribution of positron energy deposition in the oxygen gas will increase with time, which should result in a leveling off of the decrease of the $\left[\mathrm{O}_{\mathrm{I}}\right]$ doublet luminosity. This effect depends on the magnetic field strength and geometry. According to $H S T$ data, between 5.1 and $7.87 \mathrm{yr}$, the luminosity of the [O I] doublet decreased by a factor of 0.5 , which indicates a marginally slower decrease compared to the luminosity change due to the decrease of $\gamma$-ray deposition (a factor of 0.4 ). The case for which $5 \%$ positron energy is deposited in oxygen, which corresponds to equal contributions of $\gamma$-rays and positrons to the energy, predicts a drop of the [O I] doublet flux to one-half its previous value, which is more consistent with the observed evolution. However, the difference between the values predicted by the two models and the observed values is too small to permit a confident conclusion about the role of positrons in the oxygen ionization at the present time. This question may be elucidated by the future evolution of the [O I] doublet luminosity.

Another interesting by-product of the oxygen-line analysis is the upper limit on the $\gamma$-ray $(>100 \mathrm{keV})$ luminosity of a pulsar in SN 1987A imposed by the intensity of the $\left[\mathrm{O}_{\mathrm{I}}\right]$ doublet. The additional $\gamma$-ray flux related to the central source should not exceed the flux of $\gamma$-rays from $\sim 10^{-4} M_{\odot}$ of ${ }^{44} \mathrm{Ti}$, which implies that the pulsar luminosity $L_{\gamma}(>100 \mathrm{keV})<4 \times 10^{36} \mathrm{ergs} \mathrm{s}^{-1}$.

\section{THE HYDROGEN ENVELOPE}

The steady-state approximation for the treatment of the hydrogen ionization in SN 1987A already fails in the outermost layers at an age of tens of days. In the late-time nebular phase, the recombination rate $\left(\alpha n_{e}\right)$ becomes comparable to or slower than the expansion rate $\left(\alpha n_{e} t \leq 1\right)$ and deviations from the steady-state approximation become significant for the bulk of the envelope (Clayton et al. 1992; Fransson \& Kozma 1993). The present-day ionization in the hydrogen envelope is the result of the previous history of the radioactive decay in the expanding envelope and, to some extent, of the energy input at the present epoch.
The principal question is whether the latest profile and intensity of the $\mathrm{H} \alpha$ line are consistent with the standard amounts of radioactive nuclides or whether some other additional energy source (e.g., a pulsar) is needed. The answer requires solving the system of time-dependent equations of ionization and thermal balance in the freely expanding hydrogen envelope irradiated by the $\gamma$-rays emitted from decays of ${ }^{56} \mathrm{Co},{ }^{57} \mathrm{Co}$, and ${ }^{44} \mathrm{Ti}$. Such an approach was applied to the computation of the bolometric luminosity of SN 1987A over the first 4 yr by Fransson \& Kozma (1993). Here, we present results of modeling the late-time ionization and thermal structure of the SN 1987A envelope on a longer timescale in an attempt to use these quantities as a diagnostic tool for probing the primary ionization and heating sources. The equations for timedependent ionization and cooling in an expanding medium can be found elsewhere (see Dalgarno \& McCray 1972).

\subsection{Model Description}

We use a model for the density distribution of the hydrogen envelope that satisfies the basic requirements imposed by hydrodynamic models and by observational facts. The overall density distribution is assumed to be composed of three components: an inner zone $\left(v<v_{a}\right)$ of constant density $\rho_{a}$, an intermediate zone $\left(v_{a}<v<v_{b}\right)$ with some lower density $\rho_{b} \leq \rho_{a}$, and an outer zone $\left(v>v_{b}\right)$ with a decreasing outward density according to a power law $\rho=$ $\rho_{b}\left(v / v_{0}\right)^{-k}$, with $k=8$. This power is somewhat less steep than that commonly taken for the outermost layers $(k \approx 9)$ because we are interested in the lower velocity region $\left(v<7000 \mathrm{~km} \mathrm{~s}^{-1}\right)$, where $k=8$ is more appropriate. The total mass and kinetic energy are $M=14 M_{\odot}$ and $E=1.1 \times 10^{51} \mathrm{ergs}$. These choices are characterized by the parameter $E / M=0.78 \times 10^{50} \mathrm{ergs} M_{\odot}^{-1}$, which is a compromise between model 14E1 of Shigeyama \& Nomoto (1990), with the parameter $E / M=0.68 \times 10^{50} \operatorname{ergs} M_{\odot}^{-1}$, and the ratio $E / M=0.85 \times 10^{50} \operatorname{ergs} M_{\odot}^{-1}$ of the Utrobin (1993) model.

Bearing in mind that there are many unresolved problems concerning the mixing of materials with different compositions, we adopt a simple model that reflects the principal global parameters of the envelope. The model ejecta consist of a $2 M_{\odot}$ metal core, a $2 M_{\odot}$ He shell, and a $10 M_{\odot}$ hydrogen envelope, based on the model by Shigeyama \& Nomoto (1990). We assume that all the He material is completely mixed with the hydrogen envelope (10 $M_{\odot}$ ), thus producing a $12 M_{\odot}$ He-enriched hydrogen envelope that consists of 7.2 $M_{\odot}$ of hydrogen and $4.8 M_{\odot}$ of helium.

In order to be consistent with observations of the presence of $\mathrm{H}$ in the deep layers (down to $1000 \mathrm{~km} \mathrm{~s}^{-1}$, as indicated by the $\mathrm{H} \alpha$ line profile at the nebular epoch), we suggest that the hydrogen envelope is partially mixed with the metal core. To describe this mixing we assume that the 2 $M_{\odot}$ metal core is mixed macroscopically with $4 M_{\odot}$ of the hydrogen-rich envelope, thus creating $6 M_{\odot}$ of partially mixed metal core material in the constant density region $v<v_{a}$. The hydrogen was assumed to lie above the velocity $1000 \mathrm{~km} \mathrm{~s}^{-1}$. We can use the density at the level $9000 \mathrm{~km}$ $\mathrm{s}^{-1}$ found from the $\mathrm{Mg}$ II resonance doublet $\left(1.45 \times 10^{-21}\right.$ $\mathrm{g} \mathrm{cm}^{-3}$ ) to constrain this region. We find $v_{a}=2150 \mathrm{~km} \mathrm{~s}^{-1}$, as well as a density ratio in the central and intermediate zones of $\rho_{a} / \rho_{b}=3$ and a filling factor of hydrogen in the region $1000 \mathrm{~km} \mathrm{~s}^{-1}<v<v_{a}$ of 0.74 . We assume that all the 
radioactive material lies within $v<1700 \mathrm{~km} \mathrm{~s}^{-1}$, which, for our density-distribution model, gives a flux in the $847 \mathrm{keV}$ $\gamma$-ray line consistent with observational data for $t>300$ days.

The primary mechanism for hydrogen ionization at late times $(t>2 \mathrm{yr})$ is the nonthermal ionization by Compton (and secondary) electrons produced by the $\gamma$-ray scattering. The nonthermal ionization rate $\left(\right.$ in $\mathrm{cm}^{-3} \mathrm{~s}^{-1}$ ) is

$$
G_{i}=0.4\left(\epsilon_{d} / \chi_{\mathrm{H}}\right)\left(1-x^{0.2}\right),
$$

where the factor 0.4 is the branching ratio for ionization $(\mathrm{Xu}$ et al. 1992), $\epsilon_{d}$ is the energy deposition rate, $\chi_{\mathrm{H}}$ is the hydrogen ionization potential, and $x$ is the ionized fraction. The last factor in parentheses describes the fraction of the deposited energy going into ionization and excitation for the range $10^{-4}<x<0.1$; the fraction $x^{0.2}$ goes into heat. We deliberately excluded the branching of the deposited energy between $\mathrm{H}$ and $\mathrm{He}$ because the additional ionization of hydrogen by UV helium photons and He secondary electrons compensates for this power. The hydrogen recombination coefficients for the relevant temperature range are taken from Martin (1988).

During the early nebular epoch $(t<2$ yr), the photoionization of hydrogen from the second level exceeds the ionization by fast electrons from the ground level. In addition to the recombination Balmer continuum and the hydrogen two-photon radiation, we consider the photoionization by UV emission from the metal core. We assume that the luminosity of UV radiation at $\lambda<3646 \AA$ from the mixed metal core $\left(v<v_{a}\right)$ is some fraction $p$ of the total luminosity deposited in the mixed metal core. We chose $p=0.12$ based on a fit of the $\mathrm{H} \alpha$ luminosity at the early nebular phase (1-2 $\mathrm{yr})$. The average intensity of the Balmer continuum is calculated in the optically thin approximation, with the correction factor $1 /\left(1+\tau_{\mathbf{B}}\right)$ allowing for the large optical depth in the Balmer continuum $\left(\tau_{\mathbf{B}}\right)$ at early times. The population of the second level of hydrogen is determined by nonthermal ionization and excitation. We also took into account excitation of the second level by thermal electrons, which may contribute at $t<2$ years.

In the thermal balance, we included cooling by $\mathrm{H}$ ( $\mathrm{Ly} \alpha$ and two-photon radiation), [O I] $\lambda 6300, \mathrm{Mg}$ II $\lambda 2800$, and the Fe II 1.26 and $26 \mu \mathrm{m}$ lines. The Fe II $26 \mu \mathrm{m}$ transition is particularly important at an age of several years. We used Einstein coefficients for Fe II from Nussbaumer \& Storey (1988) and collision strengths from Pradhan \& Zhang
(1993). At an age of several years, when the ionization of the hydrogen envelope drops below $10^{-2}$, collisions with neutral hydrogen become the dominant mechanism for the excitation of $\mathrm{Fe}$ II fine-structure levels. The corresponding rate was taken from Dalgarno \& McCray (1972). The ionized fraction of iron in the hydrogen envelope was also calculated from a time-dependent ionization equation.

We will discuss below results obtained for two models (Table 4). Model A is the model described above; model B differs from model A by introducing an additional energy deposition rate $30 \mathrm{ergs} \mathrm{s}^{-1} \mathrm{~g}^{-1}$, which corresponds to a total power deposited in the envelope of $8.4 \times 10^{35} \mathrm{ergs}$ $\mathrm{s}^{-1}$. The adopted abundance of metals in the hydrogen envelope is one-third solar.

\subsection{Ionization, Temperature, and $\mathrm{H} \alpha$ Evolution}

For both models (Table 4) the time-dependent ionization and energy equations have been solved, beginning with the initial time $t_{0}=0.8 \mathrm{yr}$. At this epoch, the steady-state approximation is valid for most of the envelope, except for the outermost layers, with $v>7000 \mathrm{~km} \mathrm{~s}^{-1}$. Therefore, the ionization structure for $v<7000 \mathrm{~km} \mathrm{~s}^{-1}$ does not depend on the initial conditions. Determining the initial conditions for the outermost part of the envelope generally requires solving the time-dependent problem at an early epoch, beginning at $t \sim$ days, which would make the problem very complex but would not substantially change our results. We adopted the initial ionized fraction $x_{0}=10^{-4}$, which approximately corresponds to the freeze-out ionized fraction in the outer layers after the initial recombination $(t \approx 20$ days $)$.

The term "freeze-out" for the hydrogen ionization in SN 1987A must be used cautiously, because even at the present epoch complete freeze-out has not been achieved. To illustrate this point, we consider a simple analytic model for recombination in the freely expanding $(v=r / t)$ envelope with ionization sources turned off for $t>t_{1}$. The equation for the electron concentration, $n_{e}$, in the hydrogen material in that case is

$$
\frac{\partial n_{e}}{\partial t}=-\frac{3 n_{e}}{t}-\alpha n_{e}^{2},
$$

where $\alpha$ is the recombination coefficient. Using a temperature dependence of the recombination coefficient of the form $\alpha \propto T^{-\eta}$ and approximating the time dependence of temperature with the power law $T \propto t^{-\omega}$, we find the fol-

TABLE 4

\begin{tabular}{|c|c|c|}
\hline Parameter & Model A & Model B \\
\hline Total mass $\left(M_{\odot}\right) \ldots \ldots$ & 14 & 14 \\
\hline Kinetic energy $\left(10^{51}\right.$ ergs $) \ldots$ & 1.1 & 1.1 \\
\hline \multicolumn{3}{|l|}{ Mass fractions in the $\mathrm{H}$ envelope: } \\
\hline H....................... & 0.6 & 0.6 \\
\hline 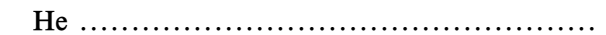 & 0.4 & 0.4 \\
\hline $\mathrm{O} \ldots \ldots$ & $2.6 \times 10^{-3}$ & $2.6 \times 10^{-3}$ \\
\hline $\mathrm{Mg} \ldots$ & $1.6 \times 10^{-4}$ & $1.6 \times 10^{-4}$ \\
\hline $\mathrm{Fe} \ldots \ldots \ldots \ldots$ & $5.4 \times 10^{-4}$ & $5.4 \times 10^{-4}$ \\
\hline \multicolumn{3}{|l|}{ Amount of radioactive nuclides $\left(M_{\odot}\right)$ : } \\
\hline 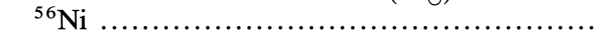 & 0.075 & 0.075 \\
\hline${ }^{57} \mathrm{Ni} \ldots \ldots \ldots \ldots$ & $2.7 \times 10^{-3}$ & $2.7 \times 10^{-3}$ \\
\hline${ }^{44} \mathrm{Ti}$. & $10^{-4}$ & $10^{-4}$ \\
\hline Additional energy deposition (ergs s${ }^{-1} \mathrm{~g}^{-1}$ )..... & 0 & 30 \\
\hline
\end{tabular}

Parameters of SN 1987A Models 


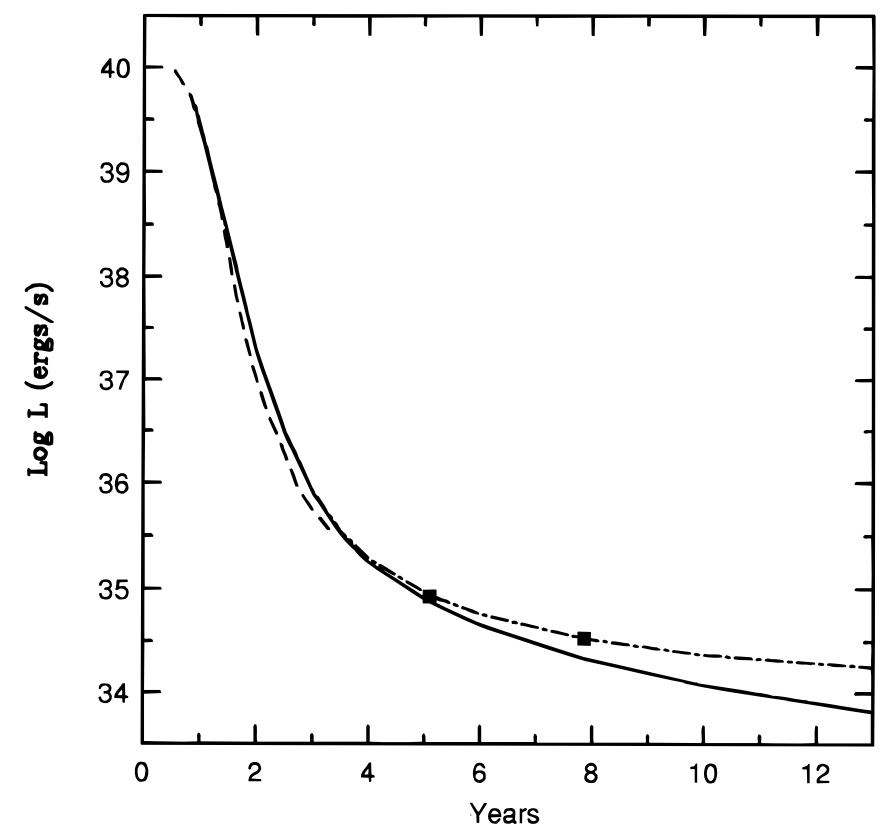

Fig. 7.-Evolution of the $\mathrm{H} \alpha$ luminosity in SN 1987A. The dashed line shows the data from Menzies (1991) and Phillips \& Williams (1991); filled squares are $H S T$ data at 5.1 and $7.87 \mathrm{yr}$. The solid line is model $\mathrm{A}$ and the dash-dotted line is model B (see text).

lowing solution for the ionization fraction $x=n_{e} / n$ :

$$
x=x_{1}\left[1+\frac{r_{1}}{2-\omega \eta}\left(1-\tau^{-2+\omega \eta}\right)\right]^{-1},
$$

where $x_{1}$ is the ionized fraction at $t=t_{1}, r_{1}=\alpha x_{1} n_{1} t_{1}$ and $\tau=t / t_{1}$ is a dimensionless time. For the particular case in which $t_{1}$ corresponds to "freeze-out", i.e., $r_{1}=1$, we find for $\eta=0.7$ and the adiabatic case $\omega=2$ that the asymptotic ionized fraction $(\tau \rightarrow \infty)$ is $x_{\infty}=0.37 x_{1}$, i.e., the ionized fraction decreases significantly after the freeze-out time. This decrease is even more dramatic for the emission measure. For $\omega>2$, which is the expected value if the cooling in lines is important, the drop of the ionized fraction at the freeze-out stage will be even higher. Because of the weak time dependence of the right-hand side of equation (12), it takes many freeze-out times to reach complete freezeout. For example, to reach the ionization fraction $x=$ $1.2 x_{\infty}$ the time required is $t=10 t_{1}$, i.e., $30 \mathrm{yr}$ for $t_{1}=3 \mathrm{yr}$. Therefore, at the present epoch the ionization of hydrogen is still changing, at least in the inner part, which contributes most of the emission measure.

The second remark concerns the relative importance of adiabatic and of line-emission cooling. At late times, when the temperature falls below $1000 \mathrm{~K}$, cooling in the Fe II 26 $\mu \mathrm{m}$ line becomes comparable to the adiabatic cooling. It is noteworthy that in the dense central region $(v<3000 \mathrm{~km}$ $\mathrm{s}^{-1}$ ), where the ionized fraction at $t>3 \mathrm{yr}$ drops far below 0.01 , the main excitation mechanism for the $\mathrm{Fe}$ II $26 \mu \mathrm{m}$ line becomes collisions with neutral hydrogen atoms. The corresponding excitation rate for the $26 \mu \mathrm{m}$ line is $q_{12}=1.44$ $\times 10^{-9} \exp \left(-E_{12} / k T\right) \mathrm{cm}^{3} \mathrm{~s}^{-1}$ (Dalgarno \& McCray 1972), which implies a critical density for collisional saturation of $n_{\mathrm{H}, \mathrm{cr}}=A_{21} / q_{21} \approx 1.2 \times 10^{6} \mathrm{~cm}^{-3}$. At $t=1500$ days, the hydrogen density in the velocity range $2000-3000 \mathrm{~km}$ $\mathrm{s}^{-1}$ is $\sim 10^{7} \mathrm{~cm}^{-3}$, so that the cooling in the $26 \mu \mathrm{m}$ line per $\mathrm{H}$ atom can be estimated as $h v A_{21}\left(n_{\mathrm{Fe}} / n_{\mathrm{H}}\right) \exp \left(-E_{12} / k T\right)$. Assuming a temperature of $700 \mathrm{~K}$ at that epoch (e.g.,
Fransson et al. 1996), we find a cooling rate per particle of $9 \times 10^{-22} \mathrm{ergs} \mathrm{s}^{-1}$. This value should be compared to the adiabatic cooling rate per particle, $3 k T / t \approx 2 \times 10^{-21} \mathrm{ergs}$ $\mathrm{s}^{-1}$, which is only twice the cooling in the $26 \mu \mathrm{m}$ line. Therefore the kinetic temperature in the hydrogen envelope after the freeze-out phase ( $t>1000$ days) should drop appreciably faster than in the pure adiabatic case. At the present time $(t=7.87 \mathrm{yr})$, the adiabatic cooling rate of the envelope gas is $4 \times 10^{36} \mathrm{ergs} \mathrm{s}^{-1}$ if $T=200 \mathrm{~K}$, and the $\mathrm{Fe}$ II $26 \mu \mathrm{m}$ emission from the envelope gas may compete with the emission from iron-rich gas.

By the present epoch $(7.87 \mathrm{yr})$, the temperature averaged over emission measure in model A falls to $130 \mathrm{~K}$, which is lower than the estimate from the Balmer continuum $(350 \pm 100 \mathrm{~K})$. However, the estimate of the observed temperature is hampered by the uncertainty in the contribution from numerous metal lines in the Balmer continuum region. We do not consider this discrepancy to show that there is some source of heating in the hydrogen envelope that is not accounted for.

The magnesium and iron in the model are singly ionized, with the ratio of neutral to ionized densities $\approx 0.1$, which is a factor of 3 larger than the estimate obtained from modeling the $2800 \AA$ magnesium emission region. However, considering that the latter estimate is uncertain by at least a factor of 2 and considering the error in extrapolating the metal recombination coefficients to the low-temperature region, the agreement may be considered to be satisfactory.

The evolution of the hydrogen ionization produced in the model can be converted into the $\mathrm{H} \alpha$ luminosity evolution. The effective recombination coefficient for $\mathrm{H} \alpha\left(\alpha_{32}\right)$, which ranges between case $\mathrm{C}$ at the early nebular epoch and case $\mathrm{B}$ at the later epoch, was approximated by a simple function of the H $\beta$ optical depth (Chugai 1990). We have taken into account the existence of the dusty core, which was turned on at $t=550$ days (Lucy et al. 1991). As in $\S 4.1$, the dusty core was described as a homogeneous sphere with an expansion velocity of $1800 \mathrm{~km} \mathrm{~s}^{-1}$ in which the dust optical depth is unity, $\tau_{d}=1$. The dust absorption reduces the apparent $\mathrm{H} \alpha$ luminosity at $t>550$ days by a factor of 1.4.

In Figure 7, the computations are compared to the observed $\mathrm{H} \alpha$ luminosity $[D=50 \mathrm{kpc}, E(B-V)=0.2]$, including $H S T$ data (at $t=5.1$ and $7.87 \mathrm{yr}$ ) and available data from earlier times $t<3.2$ yr (Menzies 1991; Phillips \& Williams 1991). The two models presented are model A, a purely radioactive source with the amounts of nuclides shown in Table 4, and model B, which differs from model A by introducing an additional deposition rate in the form of fast electrons, $q=30 \mathrm{ergs} \mathrm{s}^{-1} \mathrm{~g}^{-1}$. Model A describes the observed behavior of the $\mathrm{H} \alpha$ luminosity over a range of 5 orders of magnitude between 1 and 8 yr with a maximal deviation of a factor of 1.4. Model B removes the deficit of the model luminosity at the latest times. However, bearing in mind approximations in the model and possible errors in the spectrophotometry, we do not consider the perfect fit of model B at late times to be a proof for an additional source of energy. It shows that $8 \times 10^{35} \mathrm{ergs} \mathrm{s}^{-1}$ is the upper limit to the additional power deposited into the envelope in the form of fast electrons.

\section{CONCLUSIONS}

The UV/optical spectrum of SN 1987A at $t=7.87 \mathrm{yr}$ is characterized by the presence of strong UV resonance lines of metals, dominated by $\mathrm{Fe}$ II, $\mathrm{Mg}$ II, $\mathrm{Mg}$ I, and probably Si I 
and $\mathrm{Fe} \mathrm{I}$. In the visible wavelength region, $\mathrm{H} \alpha$ is still the strongest line. The relatively large contribution of UV Fe II resonance lines is closely connected to the low density and low kinetic temperature, which favor the high branching ratio for resonance lines as compared to subordinate lines.

Metal lines are the result of the instantaneous reemission of the present power source, dominated by the positron luminosity of ${ }^{44} \mathrm{Ti}$. The primary mechanism for photon creation is collisional excitation by fast electrons. Ionization by fast electrons with subsequent recombination may be relevant in the iron-rich zone, where the steady-state approximation is still valid. Scattering and degradation of UV resonance radiation via subordinate transitions is an important mechanism for the emission of optical radiation and produces a quasi-continuum. The hydrogen emission, dominated by $\mathrm{H} \alpha$, comes primarily from recombination and reflects the previous history of ionization, which is controlled mainly by ${ }^{56}$ Co decays.

In a broad sense, the UV/optical radiation we observe now may be defined as radioactive luminescence, which emphasizes the nonthermal nature of the radiative energy cascade, resulting in the creation of high-frequency radiation from a very cool gas. Yet it should be stressed that far-IR thermal emission $(T \approx 150 \mathrm{~K})$, both from the ironrich core and from the hydrogen-rich envelope, should dominate the bolometric luminosity.

The overall intensity of the $\mathrm{Fe}$ II lines is consistent with the presence of $\sim 1.5 \times 10^{-4} M_{\odot}$ of ${ }^{44} \mathrm{Ti}$, provided that the bulk of the positron energy is deposited into the iron-rich material. The escape of positrons from the iron-rich zone into the oxygen- and hydrogen-rich material may be inhibited by a magnetic field with strength $B \geq 5 \times 10^{-13} \mathrm{G}$. With positron trapping in the iron-rich gas, the UV/optical luminosity of the iron-rich gas will remain approximately constant during the next several years, exhibiting only a slow decrease as an increasing fraction of the energy goes into Coulomb heating as the ionization increases.

The suggested trapping of positrons in the iron-rich gas seems to agree with the intensity of the [O I $] \lambda 6300$ doublet, which is explained if the only source of energy for those lines is $\gamma$-rays from $2 \times 10^{-4} M_{\odot}$ of ${ }^{44} \mathrm{Ti}$ depositing energy in $1.5 M_{\odot}$ of oxygen-rich gas. Alternatively, the ${ }^{44} \mathrm{Ti}$ mass might be $1.5 \times 10^{-4} M_{\odot}$ for a larger oxygen mass $\left(2 M_{\odot}\right)$. An energy contribution from positrons into oxygen material is not completely ruled out, but the fraction of the positron energy going into oxygen ionization does not exceed 5\%. However, our estimates of the oxygen luminosity are based on a simplified model for the distribution of oxygen and the deposition rate. A more detailed model, which uses the observed profile of the [O I] doublet, is needed for more accurate estimates of the oxygen (or $\left.{ }^{44} \mathrm{Ti}\right)$ mass.

We did not find compelling evidence for an extra energy source in the SN 1987A ejecta apart from the standard amounts of radioactive nuclides (see Table 4). The intensity of the [O I] $\lambda 6300$ doublet sets an upper limit on the hard X-ray $/ \gamma$-ray $(h v \geq 100 \mathrm{keV})$ luminosity of a pulsar $L_{\gamma}<4$ $\times 10^{36} \mathrm{ergs} \mathrm{s}^{-1}$, which is 1 order of magnitude lower than the hard X-ray/ $\gamma$-ray luminosity of the Crab nebula.

Our time-dependent model of ionization and cooling reproduces the decrease of the $\mathrm{H} \alpha$ luminosity over 5 orders of magnitude between the ages of 1 and 8 years for the standard amount of radioactive nuclides. Although the $\mathrm{H} \alpha$ model is fairly insensitive to the mass of ${ }^{44} \mathrm{Ti}$, it is consistent with a mass $2 \times 10^{-4} M_{\odot}$ of ${ }^{44} \mathrm{Ti}$. The $H S T$ data at $t=7.87 \mathrm{yr}$ do not rule out the possibility that $\sim 10^{35} \mathrm{ergs}$ $\mathrm{s}^{-1}$ are presently deposited in the envelope in the form of fast electrons. If the source of fast electrons is the scattering of the hard X-ray $/ \gamma$-ray radiation from a central neutron star, then the required luminosity in the energy band $>100$ $\mathrm{keV}$ must be $\sim 2 \times 10^{37} \mathrm{ergs} \mathrm{s}^{-1}$. Such a luminosity can be ruled out by the upper limit on the hard X-ray/ $\gamma$-ray radiation from a central source $\left(4 \times 10^{36} \mathrm{ergs} \mathrm{s}^{-1}\right)$ imposed by the intensity of the $\left[\mathrm{O}_{\mathrm{I}}\right]$ doublet.

The estimated temperature of the iron-rich gas is $\sim 160$ $\mathrm{K}$. For the hydrogen-rich material, the time-dependent cooling and ionization model gives $T \approx 130 \mathrm{~K}$, which is somewhat lower than the observed estimate from the Balmer continuum $(350 \pm 100 \mathrm{~K})$. We do not consider this to be a significant discrepancy in view of the uncertainty of the metal-line contribution to the Balmer continuum.

We do not anticipate strong spectral evolution of SN 1987A in the UV/optical wavelength region over the next several years unless a new source of energy appears, e.g., the collision of supernova ejecta with the dense circumstellar material. Until then, we expect a slow increase in the intensity of UV lines of iron-peak elements and a slow decrease in the intensity of the fluorescent metal lines of $\mathrm{Fe}$ II and $\mathrm{Fe}$, while the total flux in lines of iron-peak elements should remain practically constant. The intensities of the [O I], Mg I], and $\mathrm{Na}$ I lines controlled by $\gamma$-ray deposition are expected to slowly decrease. Positron escape from the iron-rich material to the oxygen-rich gas may change this trend. The detection of an increase in the intensity ratios of these lines to the Fe II UV 1 and 2 lines would indicate the beginning of positron leakage from iron-rich to oxygen-rich gas.

One prediction of our model concerns the near-UV imaging of SN 1987A with HST in the F275W wavelength region. We expect that the angular radius of the outer boundary in this wavelength region to increase as $t^{(k-2) / k} \propto t^{0.75}$. The existing HST imaging data show a linear increase in the characteristic width of the image. However, this characteristic radius cannot be translated directly to the outer radius of the UV image. The only image with COSTAR (Jakobsen et al. 1994) has an outer radius consistent with our modeling of the $\mathrm{Mg}$ II line. The evolution of the outer radius of the near-UV image is valuable in yet another respect: it provides a direct method for the determination of the density gradient in the outer layers of SN 1987A.

Another prediction is that the luminosity in the $\mathrm{Fe}$ II 26

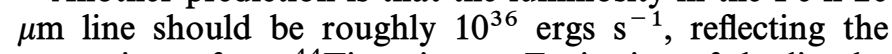
power input from ${ }^{44} \mathrm{Ti}$ positrons. Excitation of the line by neutral hydrogen collisions in the hydrogen-rich envelope may also contribute to the line flux. At a distance of $50 \mathrm{kpc}$, the expected flux is $3 \times 10^{-12} \mathrm{ergs} \mathrm{s}^{-1} \mathrm{~cm}^{-2}$, which is about an order of magnitude fainter than the observed line flux from SN 1987A during the first few 100 days (see Li et al. 1993 for references). The $26 \mu \mathrm{m}$ line should evolve slowly with time, especially if the ${ }^{44} \mathrm{Ti}$ positrons remain trapped in the iron-rich gas. This flux should be detectable with the ISO (Infrared Space Observatory) Short Wavelength Spectrometer and with future infrared observatories such as SOFIA.

We are grateful to Claes Fransson and the rest of the SINS team (David Branch, Alex Filippenko, Peter Garna- 
vich, Bruno Leibundgut, Nino Panagia, Mark Phillips, Nick Suntzeff, and J. Craig Wheeler) for their help and cooperation and to Anil Pradhan for information on atomic data. Support for this work was provided by NASA through grant No. GO-2563.01-87A from the Space Telescope Science Institute, which is operated by the Association of Universities for Research in Astronomy, Inc., under NASA contract NAS 5-26555.
Arnett, W. D., Bahcall, J. N., Kirshner, R. P., \& Woosley, S. E. 1989, ARA\&A, 27, 629

Browne, E., Firestone, R. B., \& Shirley, V. S. 1986, Table of Radioactive Isotopes (New York: Wiley-Interscience)

Chugai, N. N. 1990, Soviet Astron., 34, 96

Chugai, N. N., Danziger, I. J., Wampler, E. J., \& Wang, L. 1993, unpublished

Clayton, D. D., Leising, M. D., The, L.-S., Johnson, W. N., \& Kurfess, J. D. 1992, ApJ, 399, L141

Colgate, S. A., Petschek, A. G., \& Kriese, J. T. 1980, ApJ, 237, L81

Dalgarno, A., \& McCray, R. A. 1972, ARA\&A, 10, 375

Fransson, C., Houck, J., \& Kozma, C. 1996, in Supernovae and Supernova Remnants, ed. R. McCray \& Z. Wang (Cambridge: Cambridge Univ. Press), 211

Fransson, C., \& Kozma, C. 1993, ApJ, 408, L25

Itikawa, Y., \& Ichimura, A. 1990, J. Phys. Chem. Ref. Data, 19, 637

Jakobsen, P., Jedrzejewski, R., Macchetto, F., \& Panagia, N. 1994, ApJ, 435, L47

Keyes, C. D., et al. 1995, FOS Instrument Handbook, Version 6 (Baltimore: STScI)

Koratkar, A. 1994, in Calibrating Hubble Space Telescope, ed. J. C. Blades \& S. J. Osmer (Baltimore: STScI), 143

Kozma, C., \& Fransson, C. 1992, ApJ, 390, 602

Kurfess, J. D., et al. 1992, ApJ, 399, L137

Kurucz, R. L., \& Peytremann, E. 1975, A Table of Semiempirical gf Values, SAO Special Rep. No. 362 (Cambridge: SAO)

Laher, R. R., \& Gilmore, F. R. 1990, J. Phys. Chem. Ref. Data, 19, 277

Li, H., \& McCray, R. 1992, ApJ, 387, 309

Li, H., McCray, R., \& Sunyaev, R. A. 1993, ApJ, 419, 824

Longair, M. S. 1992, High Energy Astrophysics, 2nd ed., vol. 1 (Cambridge: Cambridge Univ. Press)

Lucy, L. B., Danziger, I. J., Gouiffes, I. J., \& Bouchet, P. 1991, in Supernovae, ed. S. E. Woosley (New York: Springer), 82

Martin, P. G. 1988, ApJS, 66, 125
McCray, R. 1993, ARA\&A, 31, 175

Meissner, J., et al. 1995, in AIP Conf. Proc. 327, Nuclei in the Cosmos III, ed. M. Busso, R. Gallino, \& C. M. Raiteri (New York: AIP), 303

Menzies, J. W. 1991, in SN 1987A and Other Supernovae, ed. I. J. Danziger \& K. Kjar (Munich: ESO), 209

Nussbaumer, H., \& Storey, P. J. 1980, A\&A, 89, 308

. 1988, A\&A, 193, 327

Panagia, N., Gilmozzi, R., Clavel, J., Barylak, M., Gonzales Riesta, R., Lloyd, C., Sanz Fernandez de Cordoba, L., \& Wamsteker, W. 1987, A\&A, 177, L25

Panagia, N., Scuderi, S., Gilmozzi, R., Challis, P. M., Garnavich, P. M., \& Kirshner, R. P. 1996, ApJ, 459, L17

Pelan, J., \& Berrington, K. A. 1997, A\&AS, 122, 177

Phillips, M. M., \& Williams, R. E. 1991, in Supernovae, ed. S. E. Woosley (New York: Springer), 36

Pradhan, A. K., \& Zhang, H. L. 1993, ApJ, 409, L77

Pun, C. S. J., et al. 1995, ApJS, 99, 223

Rosa, M., \& Mathis, J. S. 1987, ApJ, 317, 163

Rybicki, G. B., \& Hummer, D. G. 1978, ApJ, 219, 654

Seaton, M. J. 1979, MNRAS, 187, 73P

Shigeyama, T., \& Nomoto, K. 1990, ApJ, 360, 242

Shull, J. M. 1979, ApJ, 234, 761

Spyromilio, J., \& Leibundgut, B. 1995, Anglo-Aust. Obs. Newsl., 73, 1

Stathakis, R. A., Cannon, R. D., \& Spyromilio, J. 1995, Publ. Astron. Soc. Aust., 12,10

Thielemann, F.-K., Hashimoto, M., \& Nomoto, K. 1990, ApJ, 349, 222

Timmes, F. X., Woosley, S. E., Hartmann, D. H., \& Hoffman, R. D. 1996, ApJ, 464, 332

Utrobin, V. 1993, A\&A, 270, 249

Wang, L., et al. 1996, ApJ, 466, 998

Woods, D. T., Shull, J. M., \& Sarazin, C. L. 1981, ApJ, 249, 399

Woosley, S. E., Pinto, P. A., \& Hartmann, D. 1989, ApJ, 346, 395

Xu, Y., McCray, R. Oliva, E., \& Randich, S. 1992, ApJ, 386, 181 